\title{
Rapid and acute effects of estrogen on time perception in male and female rats
}

\author{
Kristen E. Pleil ${ }^{1}$, Sara Cordes ${ }^{2}$, Warren H. Meck ${ }^{3}$ and Christina L. Williams ${ }^{3}$ * \\ ${ }^{1}$ Bowles Center for Alcohol Studies, University of North Carolina, Chapel Hill, NC, USA \\ ${ }^{2}$ Department of Psychology, Boston College, Chestnut Hill, MA, USA \\ ${ }^{3}$ Department of Psychology and Neuroscience, Duke University, Durham, NC, USA
}

Edited by:

Agnes Gruart, University Pablo de

Olavide, Spain

\section{Reviewed by:}

Fuat Balci, Princeton University, USA

Angelo Santi, Wilfrid Laurier

University, Canada

*Correspondence:

Christina L. Williams, Department of Psychology and Neuroscience, Duke University, 572 Research Drive, Box 91050, GSRB-II, Room 3018, Durham, NC 27708, USA.

e-mail:williams@psych.duke.edu
Sex differences in the rapid and acute effects of estradiol on time perception were investigated in adult male and female Sprague-Dawley rats. Because estradiol has been shown to increase striatal dopamine release, it may be able to modify time perception and timed performance by increasing the speed of an internal clock in a manner similar to indirect dopamine agonists such as amphetamine and cocaine. Two groups of females (neonatally estradiol-treated/adult ovariectomized and neonatally oil-treated/adult ovariectomized) and two groups of males (neonatally castrated and adult castrated) were trained in a 2 vs. 8-s duration bisection procedure and tested using intermediate signal durations. After obtaining oil-injected baseline psychometric functions over several days, rats were administered $5 \mu \mathrm{g}$ of estradiol for 4 days and behaviorally evaluated $30 \mathrm{~min}$ following each injection. This oil-estradiol administration cycle was subsequently repeated three times following the re-establishment of baseline training. Results revealed significant sex differences in the initial baseline functions that were not modifiable by organizational hormones, with males' duration bisection functions shifted horizontally to the left of females'. Upon the first administration of estradiol, females, but not males, showed a significant, transient leftward shift in their bisection functions, indicative of an increase in clock speed. After extensive retraining in the duration bisection procedure, rats that were exposed to gonadal hormones during the first week of life showed a significant rightward shift in their bisection functions on the fourth day of estradiol administration during each cycle, suggesting a decrease in clock speed. Taken together, our results support the view that there are multiple mechanisms of estrogens' action in the striatum that modulate dopaminergic activity and are differentially organized by gonadal steroids during early brain development.

Keywords: clock speed, interval timing, duration bisection, sex differences, striatum, dopamine

\section{INTRODUCTION}

Time and space are fundamental dimensions of behavior, and competencies in these perceptual domains are rooted in neural substrates shared by many species (Gallistel, 1990; MacDonald and Meck, 2004; Buhusi and Meck, 2005; MacDonald et al., 2007). Interval timing, defined as the processing of durations in the seconds-to-minutes range, is crucial to associative learning, optimal foraging, and working memory (Gibbon et al., 1997; Meck, 2003). While considerable attention has been given to elucidating the neuroendocrine basis and underlying brain mechanisms for the different modes of spatial processing in males and females (e.g., Williams and Meck, 1991; Maguire et al., 1999; Korol, 2004), there has been comparably little work done to describe and evaluate male-female differences in temporal processing or to examine the neural and neuroendocrine underpinnings of these differences.

This gap in our understanding of interval timing is especially surprising for several reasons. A number of developmental cognitive disorders (e.g., autism, attention deficit hyperactivity disorder-ADHD, and dyslexia), and neurodegenerative disorders (e.g., Parkinson's disease - PD) have associated difficulties with aspects of temporal processing and are also known to be sexually dimorphic in both their prevalence and presentation (Teicher et al., 2000; Allman and Meck, 2011). For example, ADHD is two to ninefold more prevalent in males (Anderson et al., 1987; Arnold, 1996); however, females may be far more severely affected (Zametkin and Ernst, 1999). And, ADHD patients show impairment in time perception (Smith et al., 2002; Meck, 2005; Allman and Meck, 2011). Similarly, most reports indicate that males have a higher prevalence of PD than females (e.g., Swerdlow et al., 2001) and neurocognitive testing reveals deficits in temporal processing in these patients (Malapani et al., 1998, 2002). In both these disorders, there are underlying disturbances in dopaminergic (DA) function and targets in the basal ganglia and frontal cortex. These circuits are critically involved in temporal processing, and are organized by gonadal steroids during early development and modulated by these hormones in the adult (e.g., Xiao and Becker, 1994; Jackson et al., 2006).

It has been proposed that the neural mechanisms of interval timing are modulated by thalamo-cortico-striatal circuits, requiring DA communication between the dorsal striatum and regions 
of frontal cortex (Matell and Meck, 2000, 2004; Buhusi and Meck, 2002; MacDonald and Meck, 2004; Cheng et al., 2007b; Meck et al., 2008; Coull et al., 2011; Höhn et al., 2011). Electrophysiological studies in rats (e.g., Matell et al., 2003, 2011) and neuroimaging studies in humans (e.g., Rao et al., 2001; Hinton and Meck, 2004; Meck et al., 2008; Coull et al., 2011) have provided evidence that cortico-striatal circuits are crucially involved in the control of the internal clock. Interval timing is heavily reliant on its input from DA projections from the substantia nigra pars compacta (SNpc); lesions of the striatum (Glick and Cox, 1976; Meck, 2006b) and damaged DA projections from the $\mathrm{SNpc}$ to the striatum, as in $\mathrm{PD}$, severely impair the ability to estimate both sub-second and suprasecond durations (Malapani et al., 1998, 2002; Allman and Meck, 2011). Pharmacological manipulations of striatal DA, both systemically (Meck, 1983, 1986, 1996, 2006a; Matell et al., 2004, 2006) and intrastriatally (Neil and Herndon, 1978) alter time perception. For example, DA agonists increase the perceived amount of time that has passed, such that a given interval (e.g., $10 \mathrm{~s}$ ) is judged as being proportionally longer ( $20 \%$ increase $=12 \mathrm{~s})$, and DA antagonists decrease clock speed such that a given interval (e.g., $10 \mathrm{~s}$ ) is judged as being proportionally shorter (e.g., $20 \%$ decrease $=8 \mathrm{~s}-$ see Meck, 1996; Buhusi and Meck, 2005; Coull et al., 2011).

Numerous studies have found sex differences in striatal DA function and its response to estrogen and DA agonists (e.g., Xiao and Becker, 1994; Xiao et al., 2003; Jackson et al., 2006; Quinlan et al., 2008). Interestingly, the structure of the striatum is sexually dimorphic even during embryonic development, when the striata of females are more densely packed with DA axons and the GABAergic neurons that form striatal synapses than those of males (Ovtscharoff et al., 1992). In adulthood, females have higher basal levels of striatal DA (e.g., Walker et al., 2006) and DA transporter (DAT) mRNA (Bossé et al., 1997) than males and are more sensitive to DA agonists (Robinson et al., 1980; Beatty et al., 1982; Walker et al., 2006), even when drug concentrations in the brain are comparable (Becker et al., 1982). Gonadectomized females have higher basal levels of extracellular DA in the striatum than gonadectomized males, but there is no difference in DA uptake, suggesting possible sex differences in DA properties such as production, release, and metabolism (Castner et al., 1993). Behaviors modulated by striatal DA, such as cocaine self-administration (Hu et al., 2004; Jackson et al., 2006) and behavioral sensitization to cocaine (Hu and Becker, 2003), are greater in females than in males rats.

The underlying causes of these sex differences in striatal function may be due to organizational actions of estrogens during brain development. In both sexes, aromatase mRNA expression and activity (Küppers and Beyer, 1998), as well as both estrogen receptors (ER) $\alpha$ and $\beta$ (Küppers and Beyer, 1999) are present throughout the striatum prenatally, and these levels increase postnatally and remain throughout adulthood (but see Shughrue et al., 1997; Zhang et al., 2002). In addition, estrogen mRNA and receptor binding sites are co-localized in the female rat striatum at postnatal day 10-12 (Toran-Allerand et al., 1992). Estrogens not only increase the expression of DA receptors and their sensitivity in the striatum (Maus et al., 1990; Ferretti et al., 1992), but also influence the differentiation into (Agarti et al., 1997) and synaptogenesis of (Reisert et al., 1987; Beyer and Karolczak, 2000) dopaminergic neurons that project from the SNpc to the striatum, and the maturation of striatal GABAergic medium spiny neurons (MSNs; Stroppolo et al., 2004). Anti-estrogens administered to the neonatal male decrease adult striatal DA activity that is accompanied by decreased male sexual behavior (Gerardin et al., 2006). Taken together, these data suggest that like many other brain areas, estrogens may masculinize the striatum after its conversion from testosterone, causing the male striatum to function differently than that of the female.

In the adult, estrogens act as modulators of DA activity in the striatum through several mechanisms (Becker, 1999). Administration of estrogens directly into the striatum of adult ovariectomized rats facilitates paced mating behavior and other striatally mediated behaviors (e.g., Xiao and Becker, 1997). Ovariectomy decreases striatal DA release (Ohtani et al., 2001), D2 receptor binding (Levesque et al., 1992), and DAT activity (Le Saux and DiPaolo, 2006). While there is evidence that chronic replacement with estradiol via pulsatile or constant administration for two or more weeks restores these functions and associated behaviors, other research suggests that estrogens are only able to have these effects when administered in small, pulsatile doses over a period of several days (Becker, 1990a; Bazzett and Becker, 1994). A number of studies using DA agonists have shown that there is a rapid mechanism by which estrogens interact with striatal DA within 30 min of administration (Becker, 1990b; Xiao and Becker, 1994, 1998; Balthazart and Ball, 2006), and that this can be facilitated by priming with a longer-term physiological regimen of pulsatile estradiol (Becker and Rudick, 1999). For example, $30 \mathrm{~min}$ after a small dose of estradiol is given, the administration of amphetamine (AMPH) produces greater DA release in the striatum and rotational behavior than AMPH alone in OVX females but not castrated males (Castner et al., 1993); in addition, priming for 3 days with estradiol enhances this response to estradiol plus AMPH (Becker and Rudick, 1999). The immediate influence of estrogens on DA release are unlikely to be mediated by classical intracellular ER, but by membrane receptors that allow estrogens to rapidly alter membrane excitability. The enhanced rapid effect after several days of priming suggests that a classical estrogen receptor-mediated mechanism interacts with this rapid membrane mechanism to maximize the response to estrogen.

These data suggest that the organization and function of the striatal DA neurons that regulate interval timing may be influenced by estrogens through several different mechanisms. Therefore, adult males and females without circulating hormones may have a differentially structured and functioning striatal DA system, such that the perception of time is fundamentally different between the sexes. As a result of this sexually dimorphic organization, the clock speed of males and females may be differently altered by estradiol administration both rapidly and after priming.

Very little work has directly examined sex differences in time perception or timed performance (see MacDougall, 1904; Cheng et al., 2008a). The human literature suggests that overall, females overestimate time by approximately $10 \%$ while males do not, and females have greater variability in their time judgments (Yerkes and Urban, 1906; Eisler and Eisler, 1992; Hancock et al., 1994; Block et al., 2000; but also see Kellaris and Mantel, 1994). Sex 
differences may only be found at very short durations (Szelag et al., 2004) and only males may be able to discriminate between durations of less than $1 \mathrm{~s}$ (Rammsayer and Lustnauer, 1989; Lotze et al., 1999). However, relatively little is known about timing in the supra-seconds range.

Two recent studies have examined the role of estrogen on time perception in females. Ovariectomized rats given chronic daily injections of estradiol benzoate for 2 weeks showed a decrease in discrimination accuracy on a previously acquired task in which they had to discriminate between 2 and 8 s (Ross and Santi, 2000). This effect was present throughout both weeks of estrogen treatment and the week following, but was greatest during the second week of estrogen exposure. However, following these 2 weeks, estrogen-treated and control rats did not differ in their judgments of time intervals. While this study did not find an alteration in time perception, it is possible that this was due to the estrogen regimen that was long-term and occurred prior to time perception testing.

In contrast, ovariectomized female rats trained to lever press for food reward at 7 and $21 \mathrm{~s}$ following the onset of the "tobe-timed" signal underestimated both intervals in a proportional manner after receiving a single injection of estradiol 30 min prior to testing (Sandstrom, 2007). This time production study suggests that estradiol increases clock speed, possibly via activation of the nigrostriatal DA system, and is evidence for a rapid estrogen mechanism in the striatum that acutely alters timed performance in a manner similar to AMPH and other DA agonists (Meck, 1996; Coull et al., 2011). To date, no study has examined sex differences in time perception or their possible neuroendocrine basis. And, there has been no attempt to examine the effects of estradiol administered acutely with and without several days of pulsatile priming.

The current study aims to determine whether or not there are sex differences in time perception without circulating hormones, and if so, whether these are caused by differential brain organization due to perinatal estrogen exposure, as are many other brain structures. We will also address both the rapid (estrogen injection given $30 \mathrm{~min}$ prior to testing) and priming (three consecutive days of estrogen plus an estrogen injection given $30 \mathrm{~min}$ prior to testing) activational effects of estrogen administration on time perception in male and female rats, as others have studied striatal DA and behaviors modulated by it (Becker and Rudick, 1999). These experiments will shed light on the extent of the effects of estrogen on temporal processing and determine possible sex differences in this response to estrogen.

Although a number of different procedures can be used to access time perception in rodents (Paule et al., 1999), there are several limiting factors that must be considered in the current experiment. Because this is the first study to directly compare time perception in male and female rats, it requires a procedure in which factors such as body size, motivation, and general motor activity do not greatly affect results. Consequently, a duration bisection procedure in which rats make a choice response following the presentation of the "to-be-timed" signal allows us to measure time perception in a way that is not influenced by other performance factors related to sex differences and hormonal status (Church and Deluty, 1977; Meck, 1983; Paule et al., 1999).

\section{MATERIALS AND METHODS}

\section{ANIMALS}

Subjects were 38 rats, the offspring of 14 timed-pregnant CD Sprague-Dawley rats purchased from Charles River Laboratories (Kingston, NY, USA). Pregnant dams arrived in our colony at Duke University on their ninth day of gestation and were singly housed in individually ventilated transparent shoebox cages with corn cob bedding and ad libitum access to water and a phytoestrogen-free diet (rodent diet AIN-76A with choline chloride substituted for choline bitartrate, purchased from Dyets, American Institute of Nutrition, ICN, Nutritional Biochemical, Cleveland, OH, USA). The temperature-controlled colony room was maintained on a 12:12-h light:dark cycle with lights on at 7 a.m. daily. Upon birth, pups from all 14 litters were sexed and randomly assigned to foster mothers, and litters were culled to approximately 10 pups ( 5 females, 5 males) per dam. Pups were assigned to hormone treatment conditions within $12 \mathrm{~h}$ of birth: (a) neonatally castrated males (NCM); (b) adult castrated males (ACM); (c) neonatally estrogen-treated females (NEF); and (d) adult ovariectomized females (AOF).

After weaning on postnatal day 25, rats were housed in liketreatment groups of 4-5 for approximately 1 week, and then in pairs for the remainder of the experiment, with the same food and living conditions described for dams above. At approximately 2 months of age (postnatal day 57-58), all gonadally intact subjects were gonadectomized (subjects in all groups except NCM). Rats were approximately 4 months of age at the beginning of training. Two weeks before magazine and lever training, rats were weighed and food restricted to $85-90 \%$ of their ad libitum weight and maintained at this weight throughout the experiment to ensure responding for the food rewards. All surgeries and experiments were conducted in accordance with standard procedures approved by the Institutional Animal Care and Use Committee of Duke University.

\section{NEONATAL HORMONE MANIPULATIONS}

All male rats were anesthetized approximately $12 \mathrm{~h}$ after birth by cryoanesthesia for 3-5 min. NCMs were castrated and ACMs received sham operations, which were identical to castration in every way except for surgical removal of the testicles. Wounds were sealed with surgical glue, and pups were cleaned with alcohol and warmed before being returned to their litters. All females received subcutaneous injections of either $10 \mu \mathrm{g}$ estradiol benzoate (Steraloids, Inc., Newport, RI, USA) dissolved in $0.05 \mathrm{~mL}$ sesame oil (Sigma, St. Louis, MO, USA; NEF) or $0.05 \mathrm{~mL}$ of the oil vehicle alone (AOF) at the nape of the neck on postnatal days 0,2 , and 4 . These neonatal hormone manipulations (male castration and administration of $\mathrm{E}$ to females) have previously been shown to sex-reverse brain regions such as the hippocampus (Williams et al., 1990) and sexually dimorphic nucleus of the hypothalamus (Döhler et al., 1984). Therefore, there were four treatment groups total: Males (1) and females (2) in which we did not disrupt their normal exposure to gonadal hormones developmentally and males (3) in which we prevented exposure to gonadal testosterone postnatally and females (4) in which we exposed them to high levels of gonadal hormones postnatally. These groups allowed us to determine the contributions of postnatal organizational hormones 
and other factors (prenatal hormones and genetic factors) to be examined.

\section{ADULT HORMONE MANIPULATIONS}

At 2 months of age all rats were anesthetized for approximately $30 \mathrm{~min}$ with a cocktail of $80 \mathrm{mg} / \mathrm{kg}$ ketamine plus $10 \mathrm{mg} / \mathrm{kg}$ xylazine. ACMs underwent castrations via a medial incision in the scrotum, where testicles were exposed, tied off removed, and cauterized. The site of incision was sutured and antibiotic cream was applied. NCMs underwent sham surgeries identical to ACMs except that the testes were not tied off or removed. All females (AOF and NEF) were ovariectomized via bilateral dorsal incisions through the skin and muscle walls of the abdomen. The ovary and ovarian fat on each side of the body were exposed, tied off, and surgically removed with a scalpel. The site of removal was cauterized and carefully placed back into the abdominal cavity. The muscle wall was sutured, the skin was sutured, and antibiotic cream was applied to the wound site. These adult gonadectomies were performed in order to remove the influences of circulating gonadal hormones.

All rats received the same recovery procedures. Buprenorphine $(0.5 \mathrm{mg} / \mathrm{kg}$, i.p.) was administered at the end of all surgeries and again $12 \mathrm{~h}$ later as analgesic. Rats were kept warm on heating pads until they woke up and then returned to their home cage. Powdered food was offered in bowls on the cage floor for several days until rats were seen eating from the overhead food bin. Rats that recovered without complications from surgery were included in the subject pool for the experiment: $\mathrm{NCM}(n=8)$; ACM $(n=10)$; $\operatorname{NEF}(n=10)$; AOF $(n=10)$.

\section{APPARATUS}

All experimental data were collected in 10 standard Plexiglas and aluminum lever boxes designed for conditioning rats (Coulbourn Instruments, Allentown, PA, USA). A pellet dispenser delivered grain-based $45 \mathrm{mg}$ food pellets (Research Diets, Inc., New Brunswick, NJ, USA) to a food cup located $10 \mathrm{~cm}$ above the floor on the front wall. Two stainless-steel retractable response levers ( $4 \mathrm{~cm}$ in width) located $2 \mathrm{~cm}$ from each sidewall were horizontally placed $2.5 \mathrm{~cm}$ above the grid floor. A $2.5-\mathrm{cm}$ Sonalert calibrated to approximately $93 \mathrm{~dB}$ above background, was mounted above the food cup approximately $4 \mathrm{~cm}$ from the ceiling of the chamber was used to present auditory signals varying in duration. Each lever box was housed inside a sealed wooden sound- and lightattenuating chamber with an eyepiece viewer for observation, and was equipped with a 6 -W white house light and a $10-\mathrm{cm}$ ventilation fan. A Windows XP (Microsoft, Redmond, WA, USA) based computer system running MED-PC Version IV Research Control and Data Acquisition System software (Med Associates, St. Albans, VT, USA) attached to an electronic interface was used to control the experimental equipment and record the data.

\section{BEHAVIORAL PROCEDURES}

\section{Magazine and lever training (10 sessions)}

To prevent time of day or lever box related variation, each rat's daily experimental session occurred in the same lever box at approximately the same time each day, 7 days a week. In order to prevent distractions caused by odors from the opposite sex, especially during sessions following estradiol administration, lever boxes were assigned by genetic sex. Females (NEF and AOF) were randomly assigned to lever boxes $1-5$, and males (ACM and NCM) to lever boxes 6-10. Each of four daily sessions contained 9-10 rats, beginning at approximately 09:00, 11:00, 13:00, and 15:00, with all treatment groups represented in each session.

To train the rats to press both levers, rats received 1-h sessions of combined magazine and lever training. During each 1-h session, one food pellet was dispensed automatically once each minute and in addition, every lever press produced a pellet. To signal the start of a session, the house light turned on and both levers were retracted. Then, one lever was extended into the chamber until 10 presses were made (producing 10 food pellets), at which time this lever retracted and the other lever was extended until 10 reinforced presses were made. The lever that was extended first (left or right) was counterbalanced within each treatment group. This alternation continued for the hour, at which time the house light turned off and both levers were extended, signifying the end of the session. When all rats lever pressed at least 100 times within the hourlong session, they were transitioned to duration-discrimination training.

\section{Duration bisection procedure: two-signal training}

Rats were trained in 1-h daily sessions of duration-discrimination training in which a tone was presented for a given duration (e.g., 2 or $8 \mathrm{~s}$ ) followed by the extension of both levers. Half of the rats in each group were trained to press the left lever ("short" response) following a 2-s tone and the right lever ("long" response) following an 8-s tone. The remaining rats had this duration-lever association reversed. Each of the signal durations (2 and $8 \mathrm{~s}$ ) was randomly presented with a probability of 0.5 on each trial. The tone was presented for the selected duration, and then the levers were extended into the lever box. A response on the correct lever caused the levers to retract and a food pellet to be delivered, and a press on the incorrect lever caused the levers to retract without reinforcement. If no response was made within $10 \mathrm{~s}$, the levers retracted and the trial was considered a nonresponse. Each time the levers retracted, signifying the end of a trial, a new, randomly selected inter-trial interval (ITI) of $5 \mathrm{~s}$ plus an exponentially distributed duration with a mean of $35 \mathrm{~s}$ began.

At the end of the ITI, another signal duration was randomly selected for presentation on the next trial. A record was kept of the subject, signal duration ( 2 or $8 \mathrm{~s}$ ), lever pressed (left or right), and the latency to respond for each trial. Training occurred for 14 days, by the end of which all rats maintained a high-level of discrimination between the two anchor durations ( $>85 \%$ correct responses) for two consecutive days.

\section{Duration bisection procedure: six-signal training}

Phase 1. The conditions of two-signal training were maintained except that each of the two anchor durations ( 2 and $8 \mathrm{~s}$ ) were presented with a probability of 0.25 on each trial, and on the remaining trials, one of four probe signals of intermediate duration $(3,4,5$, and $6 \mathrm{~s})$ was presented, each with equal probability. Responses on these probe trials always caused the levers to retract without reinforcement. Again, the subject, signal duration, lever pressed, and response latency were recorded for each trial. 
The conditions of six-signal training were maintained except that rats received a subcutaneous injection of $0.1 \mathrm{~mL}$ sesame oil vehicle (Sigma, St. Louis, MO, USA) in the nape of the neck $30 \mathrm{~min}$ prior to being placed in the lever boxes for the start of the session each day for 2 days. Behavior of each rat on the second day was used as a baseline for comparison with task performance following estrogen administration.

\section{Duration bisection: estrogen cycle 1}

Baseline procedures continued, however rats received subcutaneous injections of $5 \mu \mathrm{g} 17 \beta$-estradiol (Steraloids, Inc., Newport, RI, USA) dissolved in $0.1 \mathrm{~mL}$ sesame oil in the nape of the neck $30 \mathrm{~min}$ prior to being placed in the lever boxes to start the daily session for 4 days. This 4 -day estradiol regimen has been shown to produce blood estradiol levels in the range of what is naturally produced during an estrus cycle and to increase DA activity in the striatum (Becker, 1999). Following these 4-days of estradiol administration, a 3-day "washout" period ensued, in which only the sesame oil vehicle was injected before behavioral testing.

\section{Duration bisection: seven-signal training}

Phase 2. Immediately following Phase 1, rats received vehicle injections and performed 14 daily sessions to gain experience with the duration bisection procedure. Beginning with these sessions, five intermediate signal durations were presented instead of four 2.6, 3.2, 4, 5, and $6.4 \mathrm{~s}$, which are spaced at equal logarithmic intervals between 2 and $8 \mathrm{~s}$ - so that a more accurate bisection function could be obtained in the manner of Maricq et al. (1981) and Meck $(1983,1991)$. Also, the probability of a training trial (reinforced 2 or $8 \mathrm{~s}$ tone) was reduced to $0.3(0.15$ probability of a 2 -s trial, and 0.15 probability of an 8 -s trial).

\section{Duration bisection: estrogen cycles 2-4}

After the 14 sessions of seven-signal training, three consecutive cycles of 1 day of vehicle administration followed by 4 days of estradiol administration and 9 days of vehicle washout on the seven-signal procedure ensued. For these three cycles, estrogen doses $(5 \mu \mathrm{g} \mathrm{E} 2 / 0.1 \mathrm{~mL}$ oil) were scaled to match relative average weights of each group: both genetic female groups (AOF and NEF) still received $1 \mathrm{~mL}$ of the cocktail, NCMs received $1.2 \mathrm{~mL}$ per dose, and ACMs received $1.4 \mathrm{~mL}$ per dose.

\section{DATA ANALYSIS}

The mean proportion "long" response for each signal duration was used to construct a psychometric function for each rat on each day of baseline and estrogen testing. All non-responses and responses with latencies over $4 \mathrm{~s}$ were excluded from these calculations because prior research has demonstrated that responses with long latencies are not well controlled by signal duration (e.g., Maricq et al., 1981; Maricq and Church, 1983). Days on which a rat performed poorer than $70 \%$ correct on both anchor durations were excluded and replaced with mean values of their respective groups for that session because their behavior was not reliable enough to be compared to their behavior on other sessions. This occurred on less than $10 \%$ of the sessions.

Each rat's data were fit to a three-parameter sigmoidal function in MATLAB (Mathworks - Natick, MA, USA) using a maximum likelihood method. The point of subjective equality (PSE), a measure of timing accuracy, was calculated for each subject by using this sigmoidal function to determine the signal duration that produced a "long" response $50 \%$ of the time. The difference limen (DL), a measure of variability, was calculated from the sigmoidal function by subtracting the signal duration at which $25 \%$ of responses were "long" responses from the signal duration at which $75 \%$ were "long" responses, and then dividing this value by 2 . The Weber fraction (WF), a measure of relative variability in responding, was also obtained by dividing the DL by the PSE. ANOVAs and $t$-tests were used to determine significant differences in behavior between sessions using the PSE, DL, and WF of individual participants. The alpha level was set at $p<0.05$ and all statistics were calculated using Statistica (StatSoft - Tulsa, OK, USA). In addition to the measures obtained from the sigmoidal functions, raw data points [\% "long" responses at each intermediate signal duration $3,4,5,6$, and $8 \mathrm{~s}$ (Phase 1) or 2.6, 3.2, 4, 5, and 6.4s (Phase 2)] were compared statistically to confirm the fitted PSE results. All behavioral analyses for Phase 2 were conducted using sigmoidal fits obtained by combining each rat's raw data over cycles 2,3 , and 4 because there were no differences in experimental manipulation or timing behavior across these three cycles. Analyses for estrogen effects over 4 days always included the baseline (BL), the first day of estrogen (E1), and the fourth and final day of estrogen administration (E4) because rapid estrogen effects were hypothesized to occur on E1 and acute effects on E4 within the context of the current estrogen administration paradigm (Becker and Rudick, 1999).

\section{RESULTS}

\section{PHASE 1: BASELINE DIFFERENCES}

ANOVAs were conducted in order to evaluate the betweensubjects factor of treatment group (AOF vs. NEF vs. ACM vs. NCM) on the baseline PSEs (day before estrogen administration). This test revealed a non-significant effect of treatment group, $F(3,34)=2.57, p>0.05$. In contrast, a $(2 \times 2)$ ANOVA evaluating the between-subjects factors of genetic sex and hormonal sex on the baseline PSEs revealed a main effect of genetic sex, $F(1,34)=5.84, p<0.05$, but no other reliable effects, $p s>0.05$. A post hoc $t$-test showed a significant difference of baseline PSEs between genetic males and females, $t(36)=2.32, p<0.05$, revealing the mean PSE for genetic females $(\mathrm{PSE}=4.31 \pm 0.13 \mathrm{~s})$ was significantly greater than the PSE for genetic males (PSE $=3.86 \pm 0.14 \mathrm{~s}$ ). Despite our hormonal manipulations, our results did not reveal any reliable PSE differences between hormonal males (NEF and ACM) and hormonal females (NCM and $\mathrm{AOF}$ ) at baseline. The baseline duration bisection functions for all rats divided by (a) treatment group (acute estradiol vs. oil), (b) genetic sex (male vs. female), and (c) hormonal sex (exposure to gonadal hormones or estradiol perinatally vs. no exposure to postnatal gonadal hormones) are shown in Figure 1.

To follow up these analyses of the PSE's obtained from the sigmoidal fits of the individual psychometric functions; analyses were conducted on the raw data (probability of a rat pressing the "long" lever as a function of each intermediate signal duration). Using the raw data, a $(2 \times 2 \times 3 \times 4)$ repeated measures ANOVA testing the between-subjects factors of hormonal sex and genetic sex and the 

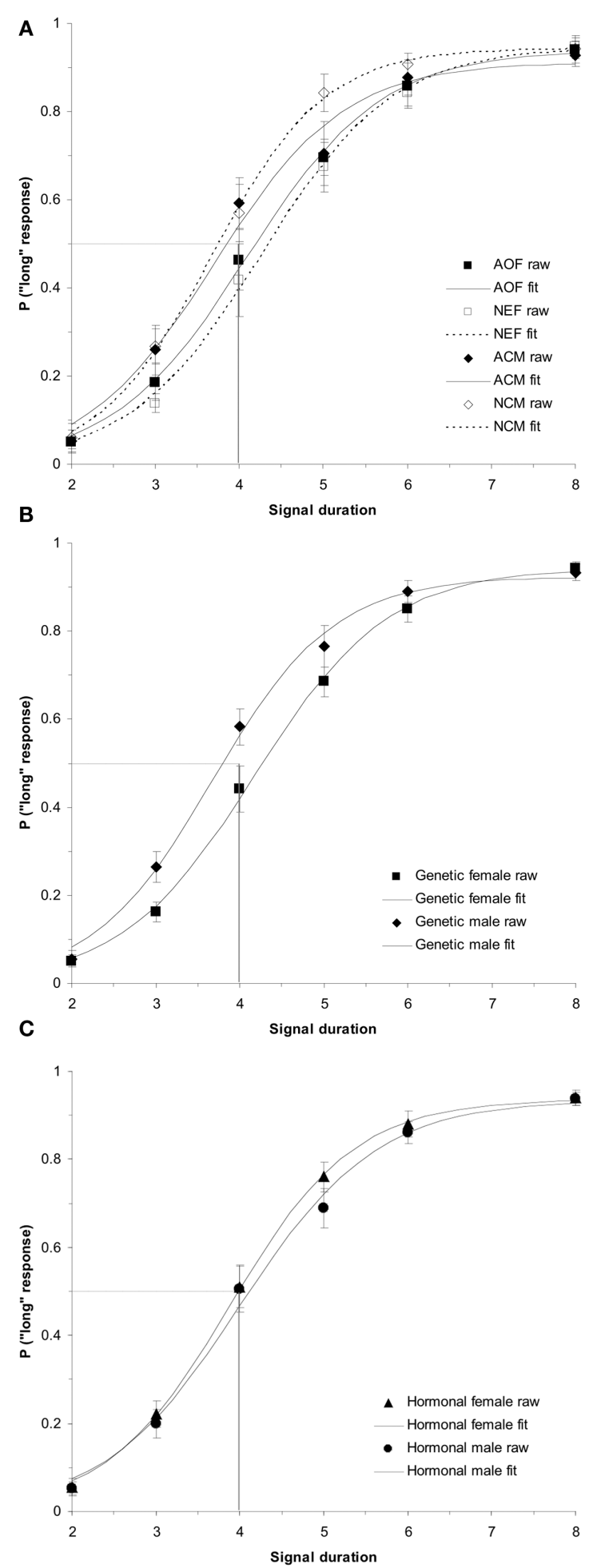

FIGURE 1 | Phase 1 duration baseline bisection function raw data and sigmoidal fits as a function of (A) treatment group (AOF, NEF, ACM, NCM); (B) genetic sex (female $=A O F+N E F$, male $=A C M+N C M)$; and (C) hormonal sex (female $=A O F+N C M$, male $=A C M+N E F)$, revealing a non-significant effect of treatment group $(p>0.05)$, a significant effect of genetic sex $(\boldsymbol{p}<\mathbf{0 . 0 5})$, and a non-significant effect of hormonal sex $(\boldsymbol{p}>\mathbf{0 . 0 5})$. Probability of a "long" response is plotted as a function of signal duration(s). within-subjects factors of test day (BL, E1, E4) and intermediate signal duration $(3,4,5$, and $6 \mathrm{~s})$ on the probability of pressing the "long" lever was conducted. It confirmed the main effect of genetic sex, $F(1,34)=5.50, p<0.05$, and no other significant effects ( $p s>0.05$ ). The main effect revealed that the females were less likely to press the "long" lever (genetic female $=0.54 \pm 0.03$ ) than the males (genetic male $=0.63 \pm 0.03$ ). Overall, these separate analyses show that genetic male PSEs and overall probability of pressing the "long" lever are significantly greater than those of genetic females, suggesting that the memory representations of the 2 and 8-s anchor duration for genetic males' are proportionally shorter than the memory representations of the same anchor durations for females' (Meck, 1983, 1991; Cheng et al., 2008b, 2011).

\section{ACUTE ESTROGEN EFFECTS}

A $(2 \times 2 \times 3)$ repeated measures ANOVA testing the betweensubjects variables of hormonal sex and genetic sex and the withinsubjects variable test day (BL, E1, E4) on the PSEs showed a significant interaction between genetic sex and test day, $F(2,68)=3.47$, $p<0.05$, and no other significant main effects or interactions ( $p s>0.05)$.

This significant interaction between genetic sex and test day was broken down into two one-way repeated measures ANOVAs for each genetic sex. First, the one-way ANOVA testing the withinsubjects variable of test day (BL, E1, E4) on PSE for the genetic females revealed a significant effect of test day, $F(2,34)=3.96$, $p<0.05$ and no other significant main effects or interactions. Follow up $t$-tests for genetic females revealed significant differences between PSEs for BL $($ mean $=4.31 \pm 0.13)$ and E1 $[$ mean $=3.96 \pm 0.14 ; t(19)=2.43, p<0.05]$, as well as E1 vs. E4 $[$ mean $=4.38 \pm 0.15 ; t(19)=2.30, p<0.05]$, but not between BL and $\mathrm{E} 4[t(19)=0.45, p>0.05]$. These results indicate that on the first day of estrogen administration, the genetic females revealed a leftward shift in the PSE (i.e., increase in clock speed), yet the PSE shifted back to baseline levels by the fourth day of estrogen administration. Secondly, the one-way ANOVA for genetic males between $\mathrm{BL}($ mean $=3.87 \pm 0.14)$, E1 (mean $=4.12 \pm 0.15)$, and $\mathrm{E} 4$ (mean $=4.09 \pm 0.15$ ) showed no reliable effect of day, $p>0.05$, suggesting that performance did not vary significantly from day to day for these subjects. Bisection functions for the baseline and the first and fourth days of estrogen administration for (a) genetic females and (b) genetic males are shown in Figure 2. The mean PSEs as a function of genetic sex for the baseline day and each of the 4-days of estrogen administration are illustrated in Figure 3A.

When a similar analysis was performed on the raw data, a $(2 \times 2 \times 3 \times 4)$ repeated measures ANOVA testing the betweensubjects variables hormonal sex and genetic sex and the withinsubjects factors of test day (BL, E1, E4) and intermediate signal duration $(3,4,5$, and $6 \mathrm{~s})$ showed a significant day $\times$ genetic sex interaction, $F(2,68)=3.00, p<0.05$, but no other significant main effects or interactions, $p s>0.05$.

In order to decompose this interaction, an ANOVA for genetic females testing the within-subjects factors of test day (BL, E1, E4) and intermediate signal duration $(3,4,5$, and $6 \mathrm{~s})$ on the raw data (probability of a "long" response for each intermediate signal duration) showed a significant effect of day, $F(2,34)=3.60$, 


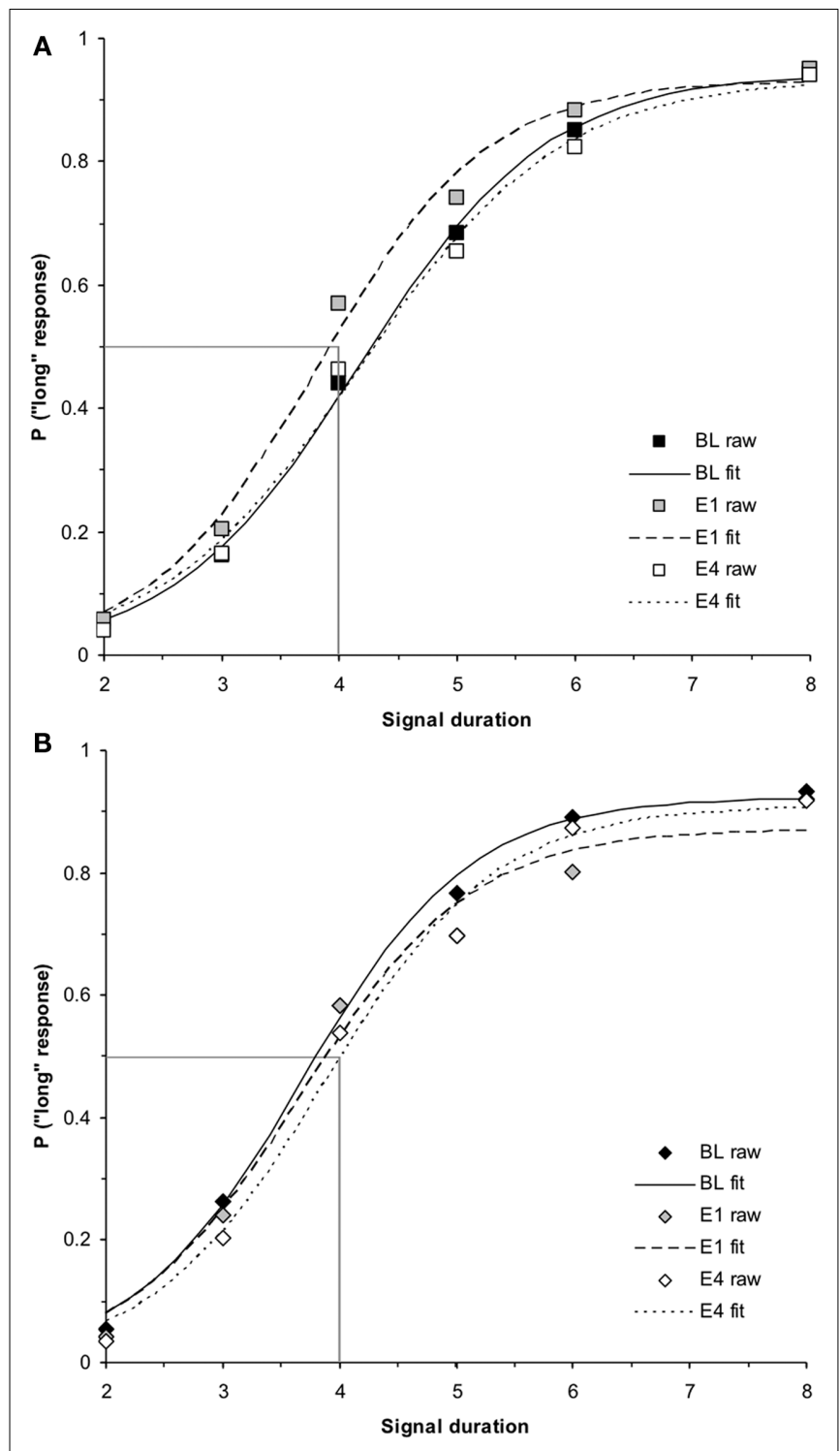

FIGURE 2 | Phase 1 duration bisection functions during baseline, E1, and E4 for (A) genetic females and (B) genetic males, revealing a significant transient leftward shift in females on E1 that returns to baseline by E4 ( $p<0.05)$, and no effect in males. Probability of a "long" response is plotted as a function of signal duration(s).

$p<0.05$ and no other significant main effects or interactions. Follow up $t$-tests showed significant differences between the overall proportion of "long" responses on $\mathrm{BL}$ (mean $=0.54 \pm 0.03$ ) and $\mathrm{E} 1$ (mean $=0.60 \pm 0.03), t(19)=2.39, p<0.05$, as well as $\mathrm{E} 1$ and $\mathrm{E} 4($ mean $=0.53 \pm 0.03), t(19)=2.25, p<0.05$, but not BL and $\mathrm{E} 4, t(19)=0.30, p>0.05$, reflecting the same pattern of results as found with the PSEs. A similar ANOVA for genetic males revealed no significant main effects or interactions, suggesting that performance on $\mathrm{BL}($ mean $=0.63 \pm 0.03)$, $\mathrm{E} 1$ (mean $=0.58 \pm 0.04)$, and $\mathrm{E} 4$ ( mean $=0.58 \pm 0.03$ ), did not differ for the males $p>0.05$. Taken together, these results show that genetic females were overall more likely to judge time intervals as "long" $30 \mathrm{~min}$ following a single injection of estradiol (increase in clock speed), and this effect

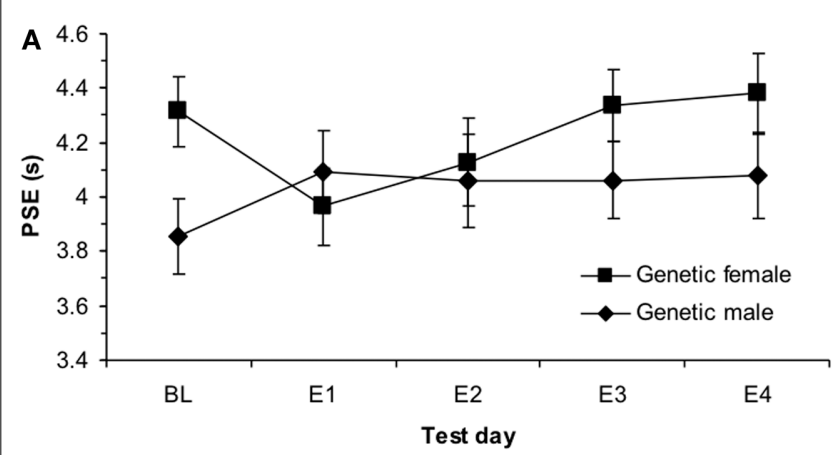

B

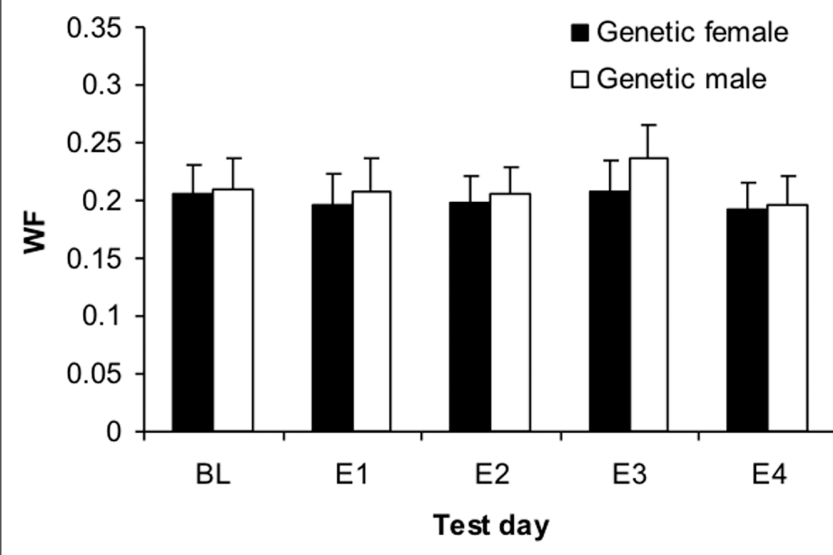

FIGURE 3 | Phase 1 sigmoidal function measures of (A) point of subjective equality (PSE) and (B) Weber fractions (WF) for genetic males and females showing a significant effect of estrogen on E1 for females in PSE $(p<0.05)$ and no effects in WF $(p>0.05)$.

returned to baseline by the fourth day of estrogen administration. Genetic males showed no changes in time perception at any time during their first 4-day exposure to estrogen. Interestingly, we again found no significant effects of hormonal sex, suggesting that genetic sex played the most important role in whether acute estrogen administration would affect time perception in Phase 1.

\section{Difference limen and Weber fraction}

In addition to analyses of the PSE, we were also interested in whether there were sex differences in variability in responding during baseline and after estradiol administration, as measured by the DL and WF. A $(2 \times 2)$ ANOVA testing the between-subjects variables of hormonal sex and genetic sex on the baseline DL revealed no significant effects or interactions, $p s>0.05$. A similar ANOVA for the WF also found no significant differences, $p s>0.05$.

A $(2 \times 2 \times 3)$ repeated measures ANOVA with betweensubjects variables of hormonal sex and genetic sex and the withinsubjects variable test day (B, E1, E4) on the DL showed no significant effects, $p s>0.05$, and a similar ANOVA for the WF also found no significant effects, $p s>0.05$. These results suggest that there were no reliable differences in variability that could account for the initial baseline differences in time perception between genetic males and females, and also that estrogen administration did not cause any changes in variability. The mean WFs as a function of genetic sex for the baseline day and each of the 4-days of estrogen 
Table 1 | Phase 1: duration bisection timing measures.

\begin{tabular}{llllll}
\hline $\begin{array}{l}\text { Genetic } \\
\text { sex }\end{array}$ & Day & PSE & DL & WF & \% “long” \\
\hline Female & BL & $4.31 \pm 0.13$ & $0.90 \pm 0.11$ & $0.21 \pm 0.03$ & $0.54 \pm 0.03$ \\
& E1 & $3.96 \pm 0.14$ & $0.78 \pm 0.11$ & $0.20 \pm 0.03$ & $0.60 \pm 0.03$ \\
& E2 & $4.13 \pm 0.16$ & $0.82 \pm 0.11$ & $0.20 \pm 0.02$ & $0.57 \pm 0.04$ \\
& E3 & $4.34 \pm 0.13$ & $0.91 \pm 0.13$ & $0.21 \pm 0.03$ & $0.53 \pm 0.02$ \\
Male & E4 & $4.38 \pm 0.15$ & $0.87 \pm 0.11$ & $0.19 \pm 0.02$ & $0.53 \pm 0.03$ \\
& BL & $3.86 \pm 0.14$ & $0.84 \pm 0.12$ & $0.21 \pm 0.03$ & $0.63 \pm 0.03$ \\
& E1 & $4.10 \pm 0.15$ & $0.89 \pm 0.12$ & $0.21 \pm 0.03$ & $0.58 \pm 0.03$ \\
& E2 & $4.06 \pm 0.17$ & $0.88 \pm 0.11$ & $0.21 \pm 0.02$ & $0.58 \pm 0.04$ \\
& E3 & $4.06 \pm 0.14$ & $1.03 \pm 0.14$ & $0.24 \pm 0.03$ & $0.57 \pm 0.03$ \\
& E4 & $4.08 \pm 0.16$ & $0.85 \pm 0.12$ & $0.20 \pm 0.02$ & $0.58 \pm 0.03$
\end{tabular}

$P S E$, point of subjective equality; $D L$, difference limen; WF, Weber fraction; $B L$, baseline day; E, estrogen day.

administration is shown in Figure 3B. Table $\mathbf{1}$ displays each of the bisection measures (e.g., PSE, DL, and WF) as a function of genetic sex for Phase 1.

\section{PHASE 2}

\section{Baseline effects}

ANOVAs comparing group baseline PSEs during Phase 2 of the experiment found no significant treatment effects, $p s>0.05$. In addition, we conducted a $(2 \times 2)$ ANOVA on baseline PSEs with between-subjects variables of hormonal sex and genetic sex, which indicated no significant main effects or interactions for PSE, $p s>0.05$. This suggests that all rats had similar PSEs and therefore had similar baseline time judgment in this procedure during Phase 2 of training. The baseline duration bisection functions for all rats divided by (a) treatment group, (b) genetic sex, and (c) hormonal sex are shown in Figure 4.

\section{Long-term estrogen effects}

A $(2 \times 2 \times 3)$ repeated measures ANOVA with between-subjects variables of hormonal sex and genetic sex and the within-subjects variable of test day (BL, E1, E4) on the PSEs revealed a significant interaction between hormonal sex and test day, $F(2,68)=3.312$, $p<0.05$, but no other significant main effects or interactions, $p s>0.05$. In order to evaluate this interaction, we conducted two follow up ANOVAs for each hormonal sex. An ANOVA on hormonal females testing the effect of test day $(B$, mean $=4.19 \pm 0.12$; $\mathrm{E} 1$, mean $=4.23 \pm 0.13$; $\mathrm{E} 4$, mean $=4.17 \pm 0.13$ ) on the PSE showed a non-significant effect of test day, $p>0.05$. In contrast, a similar ANOVA for hormonal males showed a significant effect of test day, $F(2,38)=5.39, p<0.01$. Post hoc $t$-tests on the hormonal males revealed significant differences in PSE between BL $($ mean $=4.12 \pm 0.11)$ and $\mathrm{E} 4($ mean $=4.45 \pm 0.15)$, $t(19)=-2.59, p<0.05$, as well as $\mathrm{E} 1$ (mean $=4.23 \pm 0.13$ ) and $\mathrm{E} 4, t(19)=-3.21, p<0.01$, but no significant difference between $\mathrm{B}$ and $\mathrm{E} 1, p>0.05$. Interestingly, this acute estrogen effect was modifiable by organizational hormones, as hormonal sex determined whether the estradiol affected time perception.

A $(2 \times 2 \times 3 \times 5)$ repeated measures ANOVA with betweensubjects variables of hormonal sex and genetic sex and the within-

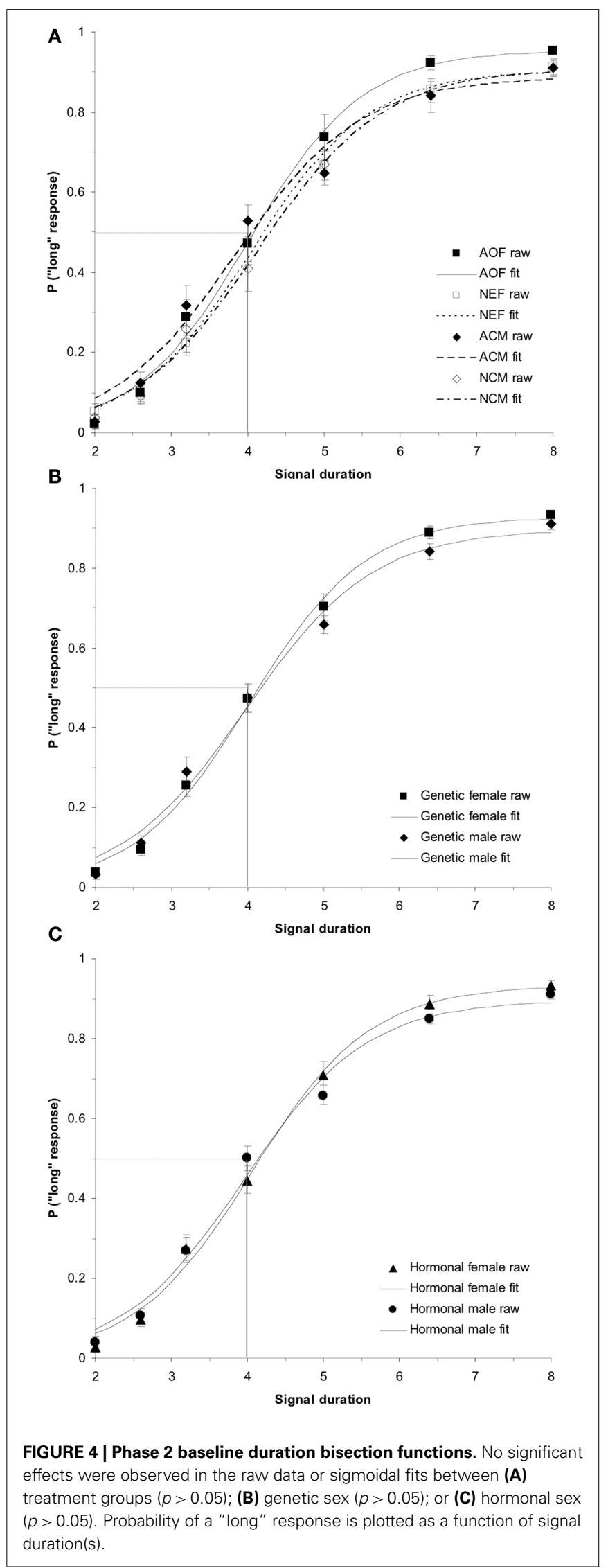


subjects variables test day $(\mathrm{B}, \mathrm{E} 1, \mathrm{E} 4)$ and intermediate signal duration $(2.6,3.2,4,5$, and $6.4 \mathrm{~s})$ on the raw data (probability of a "long" response) revealed a significant hormonal sex $\times$ test day interaction, $F(2,68)=3.15, p<0.05$, but no other reliable main effects or interactions, $p s>0.05$.

The follow up ANOVA for hormonal females testing the within-subjects factors of test day $(B$, mean $=0.48 \pm 0.02 ; E 1$, mean $=0.48 \pm 0.02 ; \mathrm{E} 4$, mean $=0.49 \pm 0.02)$ and intermediate signal duration $(2.6,3.2,4,5$, and $6.4 \mathrm{~s})$ showed no significant effects, $p>0.05$. A similar ANOVA for hormonal males showed a significant main effect of day, $F(2,38)=6.09, p<0.01$, but no other reliable effects, $p s>0.05$. Post hoc $t$-tests revealed significant differences in the probability of pressing the "long" lever between $\mathrm{B}($ mean $=0.48 \pm 0.02)$ and $\mathrm{E} 4($ mean $=0.43 \pm 0.02)$, $t(19)=2.60, p<0.05$, as well as E1 (mean $=0.47 \pm 0.02)$ and $\mathrm{E} 4$, $t(19)=4.18, p<0.01$, but not B and E1, $p>0.05$. Taken together, these results reveal that after extensive experience with the sevensignal bisection procedure, hormonal males show a rightward shift in their bisection functions (increase in PSE, slowing down of the clock) by the fourth day of estrogen administration, while hormonal females show no changes in their time perception with this estrogen administration protocol.

\section{Washout effect}

While a $(2 \times 2 \times 2)$ repeated measures ANOVA for PSE using the between-subjects factors of hormonal sex and genetic sex and the within-subjects factors of day of testing (E4, WO) did not find a significant hormonal sex $\times$ test day interaction $(p>0.05)$, a $(2 \times 2 \times 2 \times 5)$ ANOVA conducted on the probability of pressing the "long" lever for the five intermediate signal durations revealed a significant hormonal sex $\times$ test day interaction, $F(1,34)=5.19, p<0.05$, but no other significant main effects or interactions, $p s>0.05$. A post hoc $t$-test between the overall proportion of "long" responses for intermediate signal durations for hormonal males between E4 (mean $=0.43 \pm 0.02)$ and WO (mean $=0.48 \pm 0.02$ ) was significant, $t(19)=-2.82$, $p<0.05$. A similar $t$-test for hormonal females between E4 (mean $=0.49 \pm 0.02)$ and $\mathrm{WO}($ mean $=0.47 \pm 0.02)$ showed no reliable effect, $p>0.05$. These results show that the effect of 4 days of estrogen administration on hormonal males' time perception as revealed by a rightward shift in their bisection functions, is reversed once estrogen is no longer administered - duration bisection functions shift back to baseline; hormonal females maintain stable bisection functions throughout baseline, estrogen, and washout days. The duration bisection functions for BL, E1, E4, and WO for (a) hormonal females and (b) hormonal males are shown in Figure 5. The mean PSEs and WFs for hormonal females and males during baseline, 4 days of estrogen administration, and the washout day after estrogen administration are shown in Figures 6A,B, respectively.

\section{Difference limen and Weber fraction}

A $(2 \times 2)$ ANOVA for baseline DLs using the between-subjects factors of hormonal sex and genetic sex revealed no significant main effects or interactions, $p s>0.05$; a similar ANOVA for baseline WFs also showed no significant main effects or interactions, $p s>0.05$. A $(2 \times 2 \times 3)$ mixed-design ANOVA for the DL

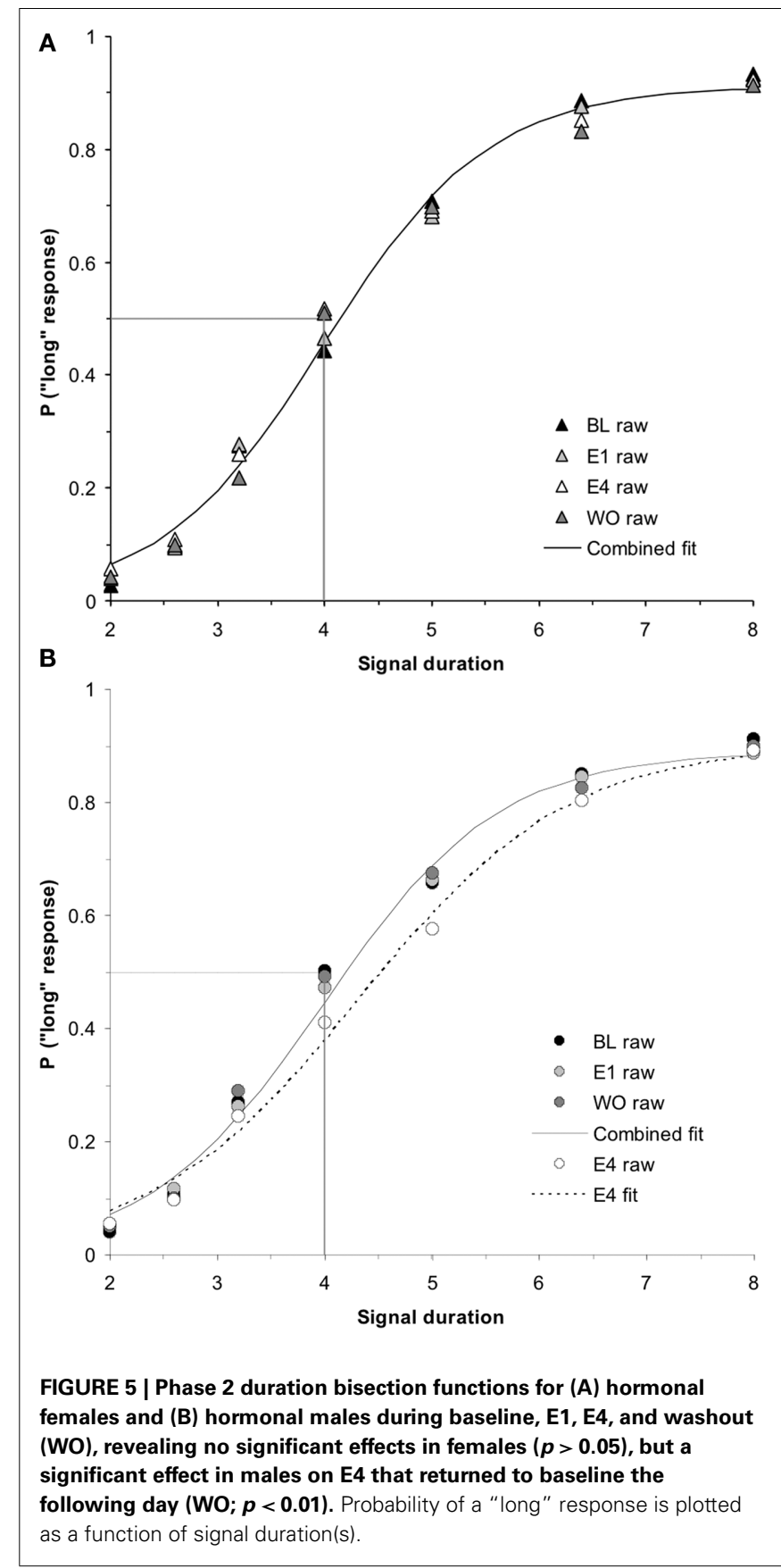

with the between-subjects factors of hormonal sex and genetic sex and within-subjects factor or test day (B, E1, E4) revealed a significant main effect of day, $F(3,102)=5.07, p<0.01$, but no other significant main effects or interactions, $p s>0.05$. A similar ANOVA for the WF also found a significant effect of day, $F(3,102)=4.03, p<0.05$, but no other significant effects, $p s>0.05$. Post hoc $t$-tests showed significant differences in the WF between $\mathrm{B}($ mean $=0.21 \pm 0.01)$ and $\mathrm{E} 4($ mean $=0.24 \pm 0.01)$, $t(37)=-2.32, p<0.05$, as well as $\mathrm{E} 1$ (mean $=0.22 \pm 0.01$ ) and $\mathrm{E} 4, t(37)=-2.182, p<0.05$, but not $\mathrm{B}$ and $\mathrm{E} 1, p>0.05$. A $(2 \times 2 \times 2)$ ANOVA for the DL using the between-subjects 


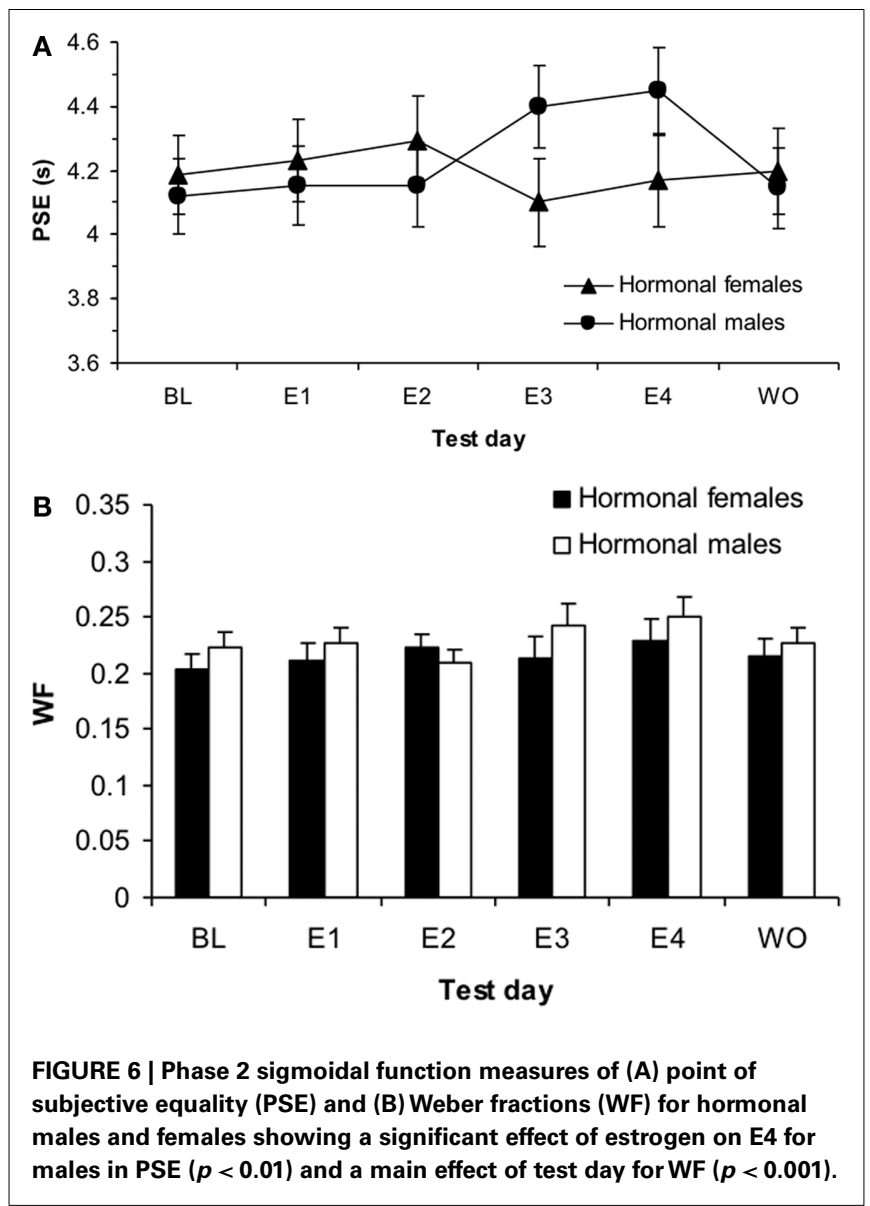

factors of hormonal sex and genetic sex and within-subjects factor test day $(\mathrm{E} 4$, mean $=1.05 \pm 0.06 ; \mathrm{WO}$, mean $=0.93 \pm 0.05)$ showed no effects, $p>0.05$. A similar ANOVA for WF (E4, mean $=0.24 \pm 0.01$; WO, mean $=0.22 \pm 0.01$ ) also found no significant effects, $p>0.05$. The mean duration bisection measures obtained for rats in each hormonal sex group are presented in Table 2. Taken together, these results suggest that there was overall more variability in timing behavior for all treatment groups on the fourth day of estrogen administration compared to baseline and the first day of estrogen.

\section{DISCUSSION}

Our duration bisection data suggest that there are fundamental sex differences in temporal processing that are sensitive to estrogen both during brain organization and in adulthood. Prior to exposure to adult circulating hormones, male and female rats showed significantly different judgments of auditory signal durations, with males' bisection functions shifted horizontally to the left of females'. This result suggests that during initial baseline training, the memory translation constant of genetic males was lower (e.g., $<1.0$ ) than that of genetic females. Such a result would occur if clock readings were "distorted" by a multiplicative translation constant $\left(\mathrm{K}^{*}\right)$ during their transfer into reference memory (Gibbon et al., 1984). $\mathrm{K}^{*}$ values less than 1.0 would produce remembered durations proportionally shorter than the physical durations and
Table 2 | Phase 2: duration bisection timing measures.

\begin{tabular}{llllll}
\hline $\begin{array}{l}\text { Hormonal } \\
\text { sex }\end{array}$ & Day & PSE & DL & WF & \% “long” \\
\hline Female & BL & $4.19 \pm 0.12$ & $0.86 \pm 0.07$ & $0.20 \pm 0.01$ & $0.48 \pm 0.02$ \\
& E1 & $4.23 \pm 0.13$ & $0.91 \pm 0.07$ & $0.21 \pm 0.01$ & $0.48 \pm 0.02$ \\
& E2 & $4.29 \pm 0.14$ & $0.96 \pm 0.07$ & $0.22 \pm 0.01$ & $0.47 \pm 0.02$ \\
& E3 & $4.10 \pm 0.14$ & $0.90 \pm 0.11$ & $0.21 \pm 0.02$ & $0.49 \pm 0.02$ \\
& E4 & $4.17 \pm 0.15$ & $0.98 \pm 0.09$ & $0.23 \pm 0.02$ & $0.49 \pm 0.02$ \\
Male & WO & $4.20 \pm 0.13$ & $0.91 \pm 0.08$ & $0.21 \pm 0.02$ & $0.47 \pm 0.02$ \\
& BL & $4.12 \pm 0.12$ & $0.92 \pm 0.07$ & $0.22 \pm 0.01$ & $0.48 \pm 0.02$ \\
& E1 & $4.15 \pm 0.12$ & $0.95 \pm 0.06$ & $0.23 \pm 0.01$ & $0.47 \pm 0.02$ \\
& E2 & $4.15 \pm 0.13$ & $0.88 \pm 0.07$ & $0.21 \pm 0.01$ & $0.47 \pm 0.02$ \\
& E3 & $4.40 \pm 0.13$ & $1.07 \pm 0.10$ & $0.24 \pm 0.02$ & $0.44 \pm 0.02$ \\
& E4 & $4.45 \pm 0.14$ & $1.10 \pm 0.09$ & $0.25 \pm 0.02$ & $0.43 \pm 0.02$ \\
& WO & $4.15 \pm 0.13$ & $0.94 \pm 0.07$ & $0.23 \pm 0.01$ & $0.48 \pm 0.02$
\end{tabular}

PSE, point of subjective equality; $D L$, difference limen; WF, Weber fraction; $B L$, baseline; E, estrogen day; WO, washout.

$\mathrm{K}^{*}$ values greater than 1.0 would produce remembered durations that are proportionally longer than the physical durations (Meck, $1983,1991)$. These types of changes in $\mathrm{K}^{*}$ are normally thought to involve alterations in cholinergic function in the cortex, but may also involve hippocampal and striatal circuits (Meck, 1996, 2002; Meck and Benson, 2002; Melgire et al., 2005; Balci et al., 2009). Moreover, estradiol administration and ovariectomy have been shown to have differential effects on cholinergic function, with estradiol administration counteracting the memory impairments produced by muscarinic receptor antagonism (Dohanich et al., 1994; Singh et al., 1994; Gibbs, 2000).

An additional finding was that following administration of a single low dose of estradiol, the clock speed of female, but not male, rats was increased such that the same signal durations were judged to be about $8 \%$ longer. These findings support and extend the observations of Sandstrom (2007), who reported that acute administration of $5 \mu \mathrm{g}$ estradiol transiently increases clock speed in female rats performing in a temporal production task. The current findings replicate this effect in rats performing a perceptual timing task (e.g., duration bisection procedure) and extend the results by showing that this "clock speed" effect of activational estradiol does not occur in males. This "clock speed" interpretation of the horizontal shifts observed in the bisection functions following estradiol administration would be greatly strengthened by the utilization of multiple sets of anchor durations - thereby allowing for the determination of whether or not the horizontal shifts are proportional to the durations being timed (Maricq et al., 1981; Meck, 1996). Interestingly, this sex difference in the effects of acute estradiol administration on temporal processing in adult rats was not modifiable by our early postnatal hormone manipulations. Neonatal gonadectomy did not feminize males, causing them to be sensitive to adult estradiol administration and show "female" effects on temporal processing, nor did early postnatal estradiol administration masculinize females to make them less sensitive to estradiol in the duration bisection procedure.

We have also uncovered a second, distinctly different effect of estrogen on temporal processing. After extensive training in our 
duration bisection procedure, chronic estrogen administration decreased clock speed of rats exposed to androgens or estrogen perinatally. Both male rats castrated as adults and females treated with estradiol postnatally judged durations as $8 \%$ shorter after 4 days of adult estradiol replacement, while this treatment had no effect on temporal processing in AOF or neonatally gonadectomized males. This effect appears to require estrogen priming for several days and concurrent estrogen activation, as duration bisection functions returned to baseline on the day following a 4-day cycle of estrogen treatment. This pattern of results reveals multiple actions of estrogen on temporal processing that are dependent, in part, on both the organizational and activational effects of estrogen.

\section{SEX DIFFERENCES IN BASELINE DURATION BISECTION FUNCTIONS}

Our data reveal that males and females have significantly different baseline functions when required to scale intermediate auditory signal durations in a duration bisection procedure. While there were no sex differences in the discrimination between the two anchor durations ( 2 vs. $8 \mathrm{~s}$ ), males judged intermediate signal durations as significantly longer than females judged them. Interestingly, in our 2 vs. $8 \mathrm{~s}$ bisection procedure, the average PSE for males was to the left and the PSE for females to the right of $4.0 \mathrm{~s}$, the geometric mean between 2.0 and $8.0 \mathrm{~s}$, which has been observed as the bisection point in many previous studies using intact male rats (Church and Deluty, 1977; Meck, 1983; Cheng et al., 2007b) and pigeons (Stubbs, 1976). However, bisection functions obtained after extensive training with intermediate signal durations revealed no sex differences in timing behavior, as males gradually shifted to the right with further training such that their baseline functions were now virtually identical to those of females by the time the second baseline was established in the current experiment. These findings are novel, as no previous work has directly compared time perception in males and females. While it is not surprising that timing functions would become more stable and closer to the theoretically predicted PSE following sufficient experience with the procedure (see Gibbon, 1981; Meck, 1983; Allen and Gibbon, 1991), it is interesting that female rats appear to reach stability in baseline functions more quickly than male rats.

There are several potential reasons why we were not able to sex-reverse the brains of male and female rats with early postnatal hormone alteration. The most likely explanation is that our early postnatal manipulations missed the sensitive period for hormonal masculinization of the relevant striatal DA-modulated circuits. However, it is possible that gonadal hormones masculinize the striatum prenatally. For example, while many hippocampaldependent behaviors, such as spatial ability, can be sex-reversed postnatally (e.g., Williams et al., 1990; Williams and Meck, 1991; Isgor and Sengelaub, 1998, 2003), the sexually dimorphic effects of stress on hippocampal-dependent trace eyeblink conditioning are organized prenatally-androgens must be removed from males early in utero in order for males to show female-typical learning effects in adulthood (Shors and Miesegaes, 2002). Moreover, both aromatase and ER $\alpha$ and $\beta$ have been found in the striatum as early as ED 14 (Küppers and Beyer, 1999). Therefore, our data suggest that if organizational hormones masculinize the striatum, much of this masculinization is likely to be occurring prenatally. This would explain why we were unable to masculinize the striata of females with early postnatal estrogen administration and to demasculinize/feminize males' striata with early postnatal castration.

Another possible explanation is that this effect is dependent on genetic factors separate from effects of gonadal hormones on brain organization. Mechanisms underlying other adaptive behaviors, such as aggression, have been shown to be influenced by genes on the sex chromosome other than the Sry gene that codes for testes development and the resulting testosterone secretions (Gatewood et al., 2006). In addition, recent evidence shows that the Sry gene on the $\mathrm{Y}$ chromosome is only expressed in TH-containing neurons in the substantia nigra ( $\mathrm{SN}$ ) and can activate $\mathrm{TH}$ production in $\mathrm{SN}$ neurons that project to the striatum independently of gonadal hormones (Dewing et al., 2006). Inactivation of Sry drastically reduces the number of $\mathrm{TH}$-containing neurons in the $\mathrm{SN}(38 \%)$ and STR $(26 \%)$ without altering the total number of neurons in the SN. Our data provide functional/behavioral support for this mechanism of DA production by showing that at least one aspect of striatal function is not modifiable by postnatal hormone manipulation, suggesting that their may be a direct genetic contribution to sexually dimorphic striatal DA function.

\section{RAPID ACTIVATIONAL EFFECT OF ESTRADIOL ON CLOCK SPEED}

Our data demonstrate that a single injection of estradiol $30 \mathrm{~min}$ prior to performance in a duration bisection procedure significantly increases the clock speed of female rats, as illustrated by a leftward shift in the PSE, without a change in discrimination accuracy. This transient shift in the psychometric function immediately following estrogen administration supports the view that estrogen can mimic some of the properties of a DA agonist (Cheng et al., 2006) in order to produce an increase in clock speed. In addition, this clock speed effect diminishes after the first exposure to estrogen which is consistent with previous work showing that rats given repeated injections of DA agonists typically adjust to changes in clock speed by renormalizing their timing functions over sessions (Meck, 1983, 1996). In accordance with these findings, our data suggest that rats quickly adapt to a new clock speed by updating the anchor durations stored in memory in order to match this change in clock speed. This rescaling of duration results in a return to baseline performance within a few sessions even though the subject remains under the influence of estrogen and its putative impact on striatal DA activity and clock speed (Coull et al., 2011).

This is the first study to examine sex differences in the classification of supra-second durations using a duration bisection procedure. Our findings show that time perception of male rats is not altered by acute estradiol administration. These results are consistent with previous work showing that male rats, unlike females, do not respond to acute estradiol with increases in striatal DA release (Becker, 1990a,b, 1999; Castner et al., 1993), ACM are less sensitive to the behavioral effects of DA agonists, and male rats achieve less sensitization to DA agonists than ovariectomized females (e.g., Hu and Becker, 2003; Hu et al., 2004).

As indicated above, our data corroborate a previous report showing that a single injection of $5 \mu \mathrm{g}$ of estradiol given to AOF $30 \mathrm{~min}$ before testing in a previously trained 7 and 21 -s peakinterval procedure results in proportional leftward shifts in peak 
time (i.e., an increase in clock speed of $4-5 \%$ ) without a change in timing accuracy (Sandstrom, 2007). Interestingly, we found that with cyclic administration, this rapid effect on clock speed occurred only during the first estradiol cycle, while Sandstrom (2007) reported an acute effect on clock speed during several subsequent estradiol administration cycles. This may be due to our 4-day cyclic administration every 2 weeks as compared to Sandstrom's daily administration of estradiol, or it may be due to the extensive training we gave rats between the first and second estrogen cycles. Our rats may not have shown behavior reflective of an acute effect because they learned to adjust their timing behavior during the first 4-day cycle of estrogen administration or because of overtraining (see Cheng et al., 2007a,c).

Both the previous (Sandstrom, 2007) and the current study support the view that estrogens may cause the rapid release of DA and thus may act much like DA agonists, causing a perceptual shift such that intervals are perceived as being proportionally shorter (Meck, 1983, 1996). While our results are consistent with the findings of Sandstrom (2007), they do not support the discrimination accuracy or lack of clock speed effects previously reported for female rats treated with chronic estradiol (Ross and Santi, 2000). Unlike our methods, which focused primarily on the rapid and acute effects of estradiol administration during testing with intermediate signal durations, Ross and Santi (2000) administered estradiol during the baseline discrimination training that occurred prior to the inclusion of intermediate signal durations. Such training would be expected to lead to the rescaling of any estradiol-induced changes in clock speed prior to testing with the intermediate signal durations and hence reduce any observed effect on the horizontal placement of the psychometric functions (Meck, 1983).

Consistent with our baseline results, we were unable to alter responsivity to acute estradiol administration in adulthood with alterations of androgens or estrogens early in development. Neonatally and ACM rats were both unresponsive to estradiol in adulthood, while females that were neonatally exposed to estrogens were just as responsive to adult estrogens as females that were not exposed to neonatal estrogens. Little previous work has examined the possibility that neonatal hormone manipulations may be able to alter sensitivity to estrogen in adulthood (e.g., Anderson et al., 2005). Therefore, it is still unclear whether or not the striatal DA system's sensitivity to estrogens and psychostimulants are determined by organizational hormones. In addition to possible prenatal hormonal organization, the mechanism of Sry in DA production may be responsible for the apparent "genetic" sex difference found in the behavioral response to acute estrogen observed in the current experiment, as genetic males and females may have different mechanisms that respond differently to estrogen administration.

\section{CHRONIC ESTRADIOL EFFECTS ON TEMPORAL PROCESSING}

When estradiol was administered to rats daily after extensive training in our duration bisection procedure, a distinctly different effect on temporal processing was uncovered. By the fourth day of estradiol administration, rats exposed to estradiol or gonadal androgens during the first week of life underestimated signal durations, while there was no effect of estradiol on adult OVX females and NCM. When no estradiol was given on a fifth day, all rats showed accurate timing with no horizontal shifts in time perception - neonatally hormone-exposed rats returned to baseline performance. These data support previous research that suggests that rapid and acute effects of estrogens in the striatum may be modulated by separate mechanisms (e.g., Becker and Rudick, 1999). However, these mechanisms have previously been described as cooperative and have been hypothesized to consist of a classical estrogen receptor-mediated mechanism that enhances the rapid excitatory mechanism in females but not males. The results of the current study suggest that the striatal DA systems of males are affected by 4 days of estradiol administration, but that this effect is in the opposite direction than the rapid effect found in females, resulting in a decrease in clock speed. Interestingly, the acute 4day mechanism was modifiable by postnatal gonadal hormones even though the rapid mechanism was not, further supporting the hypothesis that there are two distinct mechanisms of estrogen action in the striatum.

It is possible that females' internal clocks are less sensitive to fluctuations in circulating estrogen because it would not be adaptive for clock speed to change wildly in a cyclical manner along with estrogen levels. A time-dependent decrease of DAT density has been shown in the striatum and the nucleus accumbens following ovariectomy (Bossé et al., 1997). Moreover, this decrease in DAT density was not modified by estradiol administration and may have contributed to the insensitivity observed in the current study given that the DAT has been shown to be involved in the regulation of clock speed (Meck et al., 2011). In contrast, the striatal DA system of males may not be able to compensate for estradiol administration, so they may become sensitized as estrogens accumulate and down regulate DA receptors, leading to less synaptic DA activity in the striatum and a consequent decrease in clock speed. In addition, it is possible that these rats already had altered striatal DA function due to extensive training in this striatally mediated timing procedure (Cheng et al., 2007a,c).

While our results are not in complete agreement with previous interpretations of sex differences and/or estrogen effects on time perception and timed performance, they are consistent with most of the previous findings if certain procedural differences are taken into consideration (Ross and Santi, 2000; Sandstrom, 2007; Cheng et al., 2008a). Moreover, our data suggest that males may down regulate DA receptors under some conditions to compensate for stimulation of the striatal DA system by estrogen. Although this study was not designed to examine the mechanism(s) behind this acute effect in males, little previous research has examined the possible effects of estrogen on males. At this point, specific hypotheses about the mechanisms responsible for the rapid and acute effects of estrogen on clock speed remain speculative and are in need of further investigation.

\section{ACKNOWLEDGMENTS}

We would like to thank Lisa Myers and Donna Werlinig for assistance in behavioral testing and Ruey Chang and Chris MacDonald for technical guidance. 


\section{REFERENCES}

Agarti, P., Ma, Z. Q., Patrone, C., Picotti, G. B., Pellicciari, C., Bondiolotti, G., Bottone, M. G., and Maggi, A. (1997). Dopaminergic phenotype induced by oestrogens in a human neuroblastoma cell line. Eur. J. Neurosci. 9, 1008-1016.

Allen, L. G., and Gibbon, J. (1991). Human bisection at the geometric mean. Learn. Motiv. 22, 39-58.

Allman, M. J., and Meck, W. H. (2011). Pathophysiological distortions in time perception and timed performance. Brain. doi: 10.1093/brain/awr210. [Epub ahead of print].

Anderson, J. C., Williams, S., McGee, R., and Silva, P. A. (1987). DSM-III disorders in preadolescent children: prevalence in a large sample from the general population. Arch. Gen. Psychiatry 44, 69-76.

Anderson, L. I., Leipheimer, R. E., and Dluzen, D. E. (2005). Effects of neonatal and prepubertal hormonal manipulations upon estrogen neuroprotection of the nigrostriatal dopaminergic system within female and male mice. Neuroscience 130, 369-382.

Arnold, L. E. (1996). Sex differences in ADHD: conference summary. $J$. Abnorm. Child Psychol. 24, 555-569.

Balci, F., Meck, W. H., Moore, H., and Brunner, D. (2009). "Timing deficits in aging and neuropathology," in Animal Models of Human Cognitive Aging, eds J. L. Bizon and A. Wood (Totowa, NJ: Humana Press), 161-201.

Balthazart, J., and Ball, G. F. (2006). Is brain estradiol a hormone or a neurotransmitter? Trends Neurosci. 29, 241-249.

Bazzett, T. J., and Becker, J. B. (1994). Sex differences in the rapid and acute effects of estrogen on striatal D2 dopamine receptor binding. Brain Res. 637, 163-172.

Beatty, W. W., Dodge, A. M., and Traylor, K. L. (1982). Stereotyped behavior elicited by amphetamine in the rat: influences of the testes. Physiol. Behav. 28, 649-652.

Becker, J. B. (1990a). Direct effect of 17 ( $\beta$-estradiol on striatum: sex differences in dopamine release. Synapse 5, 157-164.

Becker, J. B. (1990b). Estrogen rapidly potentiates amphetamineinduced striatal dopamine release and rotational behavior during microdialysis. Neurosci. Lett. 118, 169-171.

Becker, J. B. (1999). Gender differences in dopaminergic function in striatum and nucleus accumbens. Pharmacol. Biochem. Behav.64, 803-812
Becker, J. B., Robinson, T. E., and Lorenz, K. A. (1982). Sex differences and estrous cycle variations in amphetamine-elicited rotational behavior. Eur. J. Pharmacol. 80, 65-72.

Becker, J. B., and Rudick, C. N. (1999). Rapid effects of estrogen or progesterone on the amphetamine-induced increase in striatal dopamine are enhanced by estrogen priming: a microdialysis study. Pharmacol. Biochem. Behav. 64, 53-57.

Beyer, C., and Karolczak, M. (2000). Estrogenic stimulation of neurite growth in midbrain dopaminergic neurons depends on cAMP/protein kinase A signalling. J. Neurosci. Res. 59, 107-116.

Block, R. A., Hancock, P. A., and Zakay, D. (2000). Sex differences in duration judgements: a meta-analytic review. Mem. Cognit. 28, 1333-1346.

Bossé, R., Rivest, R., and Di Paolo, T. (1997). Ovariectomy and estradiol treatment affect the dopamine transporter and its gene expression in the rat brain. Brain Res. Mol. Brain Res. 46, 343-346.

Buhusi, C. V., and Meck, W. H. (2002). Differential effects of methamphetamine and haloperidol on the control of an internal clock. Behav. Neurosci. 116, 291-297.

Buhusi, C. V., and Meck, W. H. (2005). What makes us tick? Functional and neural mechanisms of interval timing. Nat. Rev. Neurosci. 6, 755-765.

Castner, S. A., Xiao, L., and Becker, J. B. (1993). Sex differences in striatal dopamine: in vivo microdialysis and behavioral studies. Brain Res. 610, 127-134.

Cheng, R. K., Ali, Y. M., and Meck, W. H. (2007a). Ketamine "unlocks" the reduced clock-speed effect of cocaine following extended training: evidence for dopamine-glutamate interactions in timing and time perception. Neurobiol. Learn. Mem. 88, 149-159.

Cheng, R. K., Etchegaray, M., and Meck, W. H. (2007b). Impairments in timing, temporal memory, and reversal learning linked to neurotoxic regimens of methamphetamine intoxication. Brain Res. 1186, 255-266.

Cheng, R. K., Hakak, O. L., and Meck, W. H. (2007c). Habit formation and the loss of control of an internal clock: inverse relationship between the level of baseline training and the clock-speed enhancing effects of methamphetamine. Psychopharmacology (Berl.) 193, 351-362.

Cheng, R. K., Dyke, A. G., McConnell, M. W., and Meck, W. H. (2011). Categorical scaling of duration as a function of temporal context in aged rats. Brain Res. 1381, 175-186.

Cheng, R. K., MacDonald, C. J., and Meck, W. H. (2006). Differential effects of cocaine and ketamine on time estimation: implications for neurobiological models of interval timing. Pharmacol. Biochem. Behav. 85, 114-122.

Cheng, R. K., MacDonald, C. J., Williams, C. L., and Meck, W. H. (2008a). Prenatal choline supplementation alters the timing, emotion, and memory performance (TEMP) of adult male and female rats as indexed by differential reinforcement of low-rate schedule behavior. Learn. Mem. 15, 153-162.

Cheng, R. K., Scott, A. C., Penney, T. B., Williams, C. L., and Meck, W. H. (2008b). Prenatal choline availability differentially modulates timing of auditory and visual stimuli in aged rats. Brain Res. 1237, 167-175.

Church, R. M., and Deluty, M.Z. (1977). Bisection of temporal intervals. J. Exp. Psychol. Anim. Behav. Process. 3, 216-228.

Coull, J. T., Cheng, R. K., and Meck, W. H. (2011). Neuroanatomical and neurochemical substrates of timing. Neuropsychopharmacology 36, 3-25.

Dewing, P., Chiang, C. W., Sinchak, K., Sim, H., Fernagut, P. O., Kelly, S., Chesselet, M. F., Micevych, P. E., Albrecht, K. H., Harley, V. R., and Vilain, E. (2006). Direct regulation of adult brain function by the malespecific factor SRY. Curr. Biol. 16, 415-420.

Dohanich, G. P., Fader, A. J., and Javorsky, D. J. (1994). Estrogen and estrogen-progesterone treatments counteract the effect of scopolamine on reinforced T-maze alternation in female rats. Behav. Neurosci. 108, 988-992.

Döhler, K. D., Hancke, J. L., Srivastava, S. S., Hofmann, C., Shryne, J. E., and Gorski, R. A. (1984). Participation of estrogens in female sexual differentiation of the brain; neuroanatomical, neuroendocrine and behavioral evidence. Prog. Brain Res. 61, 99-117.

Eisler, H., and Eisler, A. D. (1992). Time perception: effects of sex and sound intensity on scales of subjective duration. Scand. J. Psychol. 33, 339-358.

Ferretti, C., Blengio, M., Vigna, I., Ghi, P., and Genazzani, E. (1992). Effects of estradiol on ontogenesis of striatal dopamine D1 and D2 receptor sites in male and female rats. Brain Res. 751, 212-217.

Gallistel, C. R. (1990). Representations in animal cognition: an introduction. Cognition 37, 1-22.
Gatewood, J. D., Wills, A., Shetty, S., Xu, J., Arnold, A. P., Burgoyne, P. S., and Rissman, E. F. (2006). Sex chromosome complement and gonadal sex influence aggressive and parental behaviors in mice. J. Neurosci. 26, 2335-2342.

Gerardin, D. C., Bernardi, M. M., Moreira, E. G., and Pereira, O. C. (2006). Neuroendocrine and reproductive aspects of adult male rats exposed neonatally to an antiestrogen. Pharmacol. Biochem. Behav. 83, 618-623.

Gibbon, J. (1981). On the form and location of the psychometric bisection function for time. J. Math. Psychol. 24, 58-87.

Gibbon, J., Church, R. M., and Meck, W. H. (1984). Scalar timing in memory. Ann. N. Y. Acad. Sci. 423, 52-77.

Gibbon, J., Malapani, C., Dale, C. L., and Gallistel, C. R. (1997). Toward a neurobiology of temporal cognition: advances and challenges. Curr. Opin. Neurobiol. 7, 170-184.

Gibbs, R. B. (2000). Effects of gonadal hormone replacement on measures of basal forebrain cholinergic function. Neuroscience 101, 931-938.

Glick, S. D., and Cox, R. D. (1976). Differential effects of unilateral and bilateral caudate lesions on side preferences and timing behavior in rats. J. Comp. Physiol. Psychol. 90, 528-535.

Hancock, P. A., Arthur, E. J., Chrysler, S. T., and Lee, J. (1994). The effects of sex, target duration, and illumination on the production of time intervals. Acta Psychol. (Amst.) 86, 57-67.

Hinton, S. C., and Meck, W. H. (2004). Frontal-striatal circuitry activated by human peak-interval timing in the supra-seconds range. Brain Res. Cogn. Brain Res. 21, 171-182.

Höhn, S., Dallérac, G., Faure, A., Urbach, Y., Nguyen, H. P., Riess, O., von Hörsten, S., Le Blanc, P., Desvignes, N., El Massioui, N., Brown, B. L., and Doyère, V. (2011). Behavioral and in vivo electrophysiological evidence for presymptomatic alteration of prefronto-striatal processing in the transgenic rat model for Huntington disease. J. Neurosci. 31, 8986-8997.

Hu, M., and Becker, J. B. (2003). Effects of sex and estrogen on behavioral sensitization to cocaine in rats. $J$. Neurosci. 23, 693-699.

Hu, M., Crombag, H. S., Robinson, T. E., and Becker, J. B. (2004). Biological basis of sex differences in the propensity to self-administer cocaine. Neuropsychopharmacology 29, 81-85.

Isgor, C., and Sengelaub, D. R. (1998). Prenatal gonadal steroids affect adult spatial behavior, CA1 and CA3 
pyramidal cell morphology in rats. Horm. Behav. 34, 183-198.

Isgor, C., and Sengelaub, D. R. (2003). Effects of neonatal gonadal steroids on adult CA3 pyramidal neuron dendritic morphology and spatial memory in rats. J. Neurobiol. 55, 179-190.

Jackson, L. R., Robinson, T. E., and Becker, J. B. (2006). Sex differences and hormonal influences on acquisition of cocaine self-administration in rats. Neuropsychopharmacology 31, 129-138.

Kellaris, J. J., and Mantel, S. P. (1994). The influence of mood and gender on consumers' time perceptions. Adv. Consum. Res. 21, 514-518.

Korol, D. L. (2004). Role of estrogen in balancing contributions from multiple memory systems. Neurobiol. Learn. Mem. 82, 309-323.

Küppers, E., and Beyer, C. (1998). Expression of aromatase in the embryonic and postnatal mouse striatum. Brain Res. Mol. Brain Res. 63, 184-188.

Küppers, E., and Beyer, C. (1999). Expression of estrogen receptoralpha and beta mRNA in the developing and adult mouse striatum. Neurosci. Lett. 276, 95-98.

Le Saux, M., and DiPaolo, T. (2006). Influence of oestrogenic compounds on monoamine transporters in rat striatum. J. Neuroendocrinol. 18, 25-32.

Levesque, D., Gagne, B., Barden, N., and DiPaolo, T. (1992). Chronic estradiol treatment increases anterior pituitary but not striatal D2 dopamine receptor mRNA levels in rats. $\mathrm{Neu}$ rosci. Lett. 140, 5-8.

Lotze, M., Wittmann, M., von Steinbuchel, N., Poppel, E., and Roenneberg, T. (1999). Daily rhythm of temporal resolution in the auditory system. Cortex 35, 89-100.

MacDonald, C. J., Cheng, R. K., Williams, C. L., and Meck, W. H. (2007). Combined organizational and activational effects of short and long photoperiods on spatial and temporal memory in rats. Behav. Processes 74, 226-233.

MacDonald, C. J., and Meck, W. H. (2004). Systems-level integration of interval timing and reaction time. Neurosci. Biobehav. Rev. 28, 747-769.

MacDougall, R. (1904). Sex differences in the sense of time. Science 19, 707-708.

Maguire, E. A., Burgess, N., and O’Keefe, J. (1999). Human spatial navigation: cognitive maps, sexual dimorphism, and neural substrates. Curr. Opin. Neurobiol. 9, 171-177.

Malapani, C., Deweer, B., and Gibbon, J. (2002). Separating storage from retrieval dysfunction of temporal memory in Parkinson's disease. J. Cogn. Neurosci. 14, 311-322.

Malapani, C., Rakitin, B., Levy, R., Meck, W. H., Deweer, B., Dubois, B., and Gibbon, J. (1998). Coupled temporal memories in Parkinson's disease: a dopamine-related dysfunction. J. Cogn. Neurosci. 10, 316-331.

Maricq, A. V., and Church, R. M. (1983). The differential effects of haloperidol and methamphetamine on time estimation in the rat. Psychopharmacology (Berl.) 79, 10-15.

Maricq, A. V., Roberts, S., and Church, R. M. (1981). Methamphetamine and time estimation. J. Exp. Psychol. Anim. Behav. Process. 7, 18-30.

Matell, M. S., Bateson, M., and Meck, W. H. (2006). Single-trials analyses demonstrate that increases in clock speed contribute to the methamphetamine-induced horizontal shifts in peak-interval timing functions. Psychopharmacology (Berl.) 188, 201-212.

Matell, M. S., King, G. R., and Meck, W. H. (2004). Differential adjustment of interval timing by the chronic administation of intermittent or continuous cocaine. Behav. Neurosci. 118, 150-156.

Matell, M. S., and Meck, W. H. (2000). Neuropsychological mechanisms of interval timing behavior. Bioessays 22, 94-103.

Matell, M. S., and Meck, W. H. (2004). Cortico-striatal circuits and interval timing: coincidence-detection of oscillatory processes. Brain Res. Cogn. Brain Res. 21, 139-170.

Matell, M.S., Meck, W. H., and Nicolelis, M. A. (2003). Interval timing and the encoding of signal duration by ensembles of cortical and striatal neurons. Behav. Neurosci. 117, 760-773.

Matell, M. S., Shea-Brown, E., Gooch, C., Wilson, A. G., and Rinzel, J. (2011). A heterogeneous population code for elapsed time in rat medial agranular cortex. Behav. Neurosci. 125, 54-73.

Maus, M., Premont, J., and Glowinski, J. (1990). In vitro effects of 17 betaoestradiol on the sensitivity of receptors coupled to adenylate cyclase on striatal neurons in primary culture. Ciba Found. Symp. 153, 145-153.

Meck, W. H. (1983). Selective adjustment of the speed of internal clock and memory processes. J. Exp. Psychol. Anim. Behav. Process. 9, 171-201.

Meck, W. H. (1986). Affinity for the dopamine D2 receptor predicts neuroleptic potency in decreasing the speed of an internal clock. Pharmacol. Biochem. Behav. 25, 1185-1189.

Meck, W. H. (1991). Modality-specific circadian rhythmicities influence mechanisms of attention and memory for interval timing. Learn. Motiv. 22, 153-179.

Meck, W. H. (1996). Neuropharmacology of timing and time perception. Brain Res. Cogn. Brain Res. 3, 227-242.

Meck, W. H. (2002). Choline uptake in the frontal cortex is proportional to the absolute error of a temporal memory translation constant in mature and aged rats. Learn. Motiv. 33, 88-104.

Meck, W. H. (2003). Functional and Neural Mechanisms of Interval Timing. Boca Raton, FL: CRC Press LLC.

Meck, W. H. (2005). Neuropsychology of timing and time perception. Brain Cogn. 58, 1-8.

Meck, W. H. (2006a). Frontal cortex lesions eliminate the clock speed effect of dopaminergic drugs on interval timing. Brain Res. 1108 157-167.

Meck, W. H. (2006b). Neuroanatomical localization of an internal clock: a functional link between mesolimbic, nigrostriatal, and mesocortical dopaminergic systems. Brain Res. 1109, 93-107.

Meck, W. H., and Benson, A. M. (2002). Dissecting the brain's internal clock: how frontal-striatal circuitry keeps time and shifts attention. Brain Cogn. 48,195-211.

Meck, W. H., Cheng, R. K., MacDonald, C. J., Gainetdinov, R. R., Caron, M. G., and Çevik, M. Ö. (2011). Gene-dose dependent effects of methamphetamine on interval timing in dopamine-transporter knockout mice. Neuropharmacology. doi: 10.1016/j.neuropharm.2011.01.042. [Epub ahead of print].

Meck, W. H., Penney, T. B., and Pouthas, V. (2008). Cortico-striatal representation of time in animals and humans. Curr. Opin. Neurobiol. 18, 145-152.

Melgire, M., Ragot, R., Samson, S., Penney, T. B., Meck, W. H., and Pouthas, V. (2005). Auditory/visual duration bisection in patients with left or right medial-temporal lobe resection. Brain Cogn. 58, 119-124.

Neil, D. B., and Herndon, J. G. Jr. (1978). Anatomical specificity within rat striatum for the dopaminergic modulation of DRL responding and activity. Brain Res. 153, 529-538.

Ohtani, J., Nomoto, M., and Douchi, T. (2001). Chronic estrogen treatment replaces striatal dopaminergic function in ovariectomized rats. Brain Res. 900, 163-168.

Ovtscharoff, W., Eusterschulte, B., Zienecker, R., Reisert, I., and Pilgrim, C.
(1992). Sex differences in densities of dopaminergic fibers and GABAergic neurons in the prenatal rat striatum. J. Comp. Neurol. 323, 299-304.

Paule, M. G., Meck, W. H., McMillan, D. E., Bateson, M., Popke, E. J., Chelonis, J. J., and Hinton, S. C. (1999). The use of timing behaviors in animals and humans to detect drug and/or toxicant effects. Neurotoxicol. Teratol. 21, 491-502.

Quinlan, M. G., Hussain, D., and Brake, W. G. (2008). Use of cognitive strategies: the role of estradiol and its interaction with dopamine. Horm. Behav. 53, 185-191.

Rammsayer, T., and Lustnauer, S. (1989). Sex differences in time perception. Percept. Mot. Skills 68, 195-198.

Rao, S. M., Mayer, A. R., and Harrington, D. L. (2001). The evolution of brain activation during temporal processing. Nat. Neurosci. 4, 317-323.

Reisert, I., Han, V., Lieth, E., ToranAllerand, D., Pilgrim, C., and Lauder, J. (1987). Sex steroids promote neurite growth in mesencephalic tyrosine hydroxylase immunoreactive neurons in vitro. Int. J. Dev. Neurosci. 5, 91-98.

Robinson, T. E., Becker, J. B., and Ramirez, V. D. (1980). Sex differences in amphetamine-elicited rotational behavior and the lateralization of striatal dopamine in rats. Brain Res. Bull. 5, 539-545.

Ross, L., and Santi, A. (2000). The effects of estrogen on temporal and numerical processing in ovariectomized female rats. Psychobiology (Austin, Tex.) 28, 394-405.

Sandstrom, N. J. (2007). Estradiol modulation of the speed of an internal clock. Behav. Neurosci. 121, 422-432.

Shors, T. J., and Miesegaes, G. (2002). Testosterone in utero and at birth dictates how stressful experience will affect learning in adulthood. Proc. Natl. Acad. Sci. U.S.A. 99, 13955-13960.

Shughrue, P. J., Lane, M. V., and Merchenthaler, I. (1997). Comparative distribution of estrogen receptor-alpha and -beta mRNA in the rat central nervous system. J. Comp. Neurol. 388, 507-525.

Singh, M., Meyer, E. M., Millard, W. J., and Simpkins, J. W. (1994). Ovarian steroid deprivation results in a reversible learning impairment and compromised cholinergic function in female Sprague-Dawley rats. Brain Res. 644, 305-312.

Smith, A., Taylor, E., Rogers, J. W., Newman, S., and Rubia, K. (2002) Evidence for a pure time perception deficit in children with 
ADHD. J. Child Psychol. Psychiatry $43,529-542$.

Stroppolo, A., Tian, C., Guinea, B., Olm, V., Sheffield, R., Sommer, J., and Ehrlich, M. E. (2004). 17( $\beta$-estradiol promotes striatal medium size spiny neuronal maturation in vitro. $\mathrm{Neu}$ roendocrinology 79, 259-267.

Stubbs, D. A. (1976). Scaling of stimulus duration by pigeons. J. Exp. Anal. Behav. 26, 15-25.

Swerdlow, R. H., Parker, W. D. Jr., Currie, L. J., Bennett, J. P. Jr., Harrison, M. B., Trugman, J. M., and Wooten, G. F. (2001). Gender ratio differences between Parkinson's disease patients and their affected relatives. Parkinsonism Relat. Disord. 7 , 129-133.

Szelag, E., Kanabus, M., Kolodziejczyk, I., Kowalska, J., and Szuchnik, J. (2004). Individual differences in temporal information processing in humans. Acta Neurobiol. Exp. (Wars.) 64, 349-366.

Teicher, M. H., Anderson, C. M., Polcari, A., Glod, C. A., Maas, L. C., and Renshaw, P. F. (2000). Functional deficits in basal ganglia of children with attention-deficit/hyperactivity disorder shown with functional magnetic resonance imaging relaxometry. Nat. Med. 6, 470-473.

Toran-Allerand, C. D., Miranda, R. C., Hochberg, R. B., and MacLusky, N. J. (1992). Cellular variations in estrogen receptor mRNA translation in the developing brain: evidence from combined [125I] estrogen autoradiography and non-isotopic in situ hybridization histochemistry. Brain Res. 576, 25-41.

Walker, Q. D., Ray, R., and Kuhn, C. M. (2006). Sex differences in neurochemical effects of dopaminergic drugs in rat striatum. Neuropsychopharmacology 31, 1193-1202.

Williams, C. L., Barnett, A. M., and Meck, W. H. (1990). Organizational effects of early gonadal secretions on sexual differentiation in spatial memory. Behav. Neurosci. 104, 84-97.

Williams, C. L., and Meck, W. H. (1991). The organizational effects of gonadal steroids on sexually dimorphic spatial ability. Psychoneuroendocrinology 16, 155-176.

Xiao, L., and Becker, J. B. (1994). Quantitative microdialysis determination of extracellular striatal dopamine concentration in male and female rats: effects of estrous cycle and gonadectomy. Neurosci. Lett. 180 155-158.

Xiao, L., and Becker, J. B. (1997). Hormonal activation of the striatum and the nucleus accumbens modulates paced mating behavior in the female rat. Horm. Behav. 32, 114-124.

Xiao, L., and Becker, J. B. (1998). Effects of estrogen agonists on amphetamine-stimulated striatal dopamine release. Synapse 29, 379-391.

Xiao, L., Jackson, L. R., and Becker, J. B. (2003). The effect of estradiol in the striatum is blocked by ICI 182,780 but not tamoxifen: pharmacological and behavioral evidence. Neuroendocrinology 77 , 239-245.

Yerkes, R. M., and Urban, F. M. (1906). Time-estimation in its relations to sex, age, and physiological rhythms. Harvard Psychol. Stud. 2, 405-430.

Zametkin, A. J., and Ernst, M. (1999). Problems in the management of attention-deficit-hyperactivity disorder. N. Engl. J. Med. 340, 40-46.

Zhang, J.-Q., Cai, W.-Q., Zhou, D.-S., and Su, B.-Y. (2002). Distribution differences of estrogen receptor beta immunoreactivity in the brain of adult male and female rats. Brain Res. 935, 73-80.

Conflict of Interest Statement: The authors declare that the research was conducted in the absence of any commercial or financial relationships that could be construed as a potential conflict of interest.

Received: 29 June 2011; accepted: 28 September 2011; published online: 14 October 2011.

Citation: Pleil KE, Cordes S, Meck WH and Williams CL (2011) Rapid and acute effects of estrogen on time perception in male and female rats. Front. Integr. Neurosci. 5:63. doi: 10.3389/fnint.2011.00063

Copyright (0) 2011 Pleil, Cordes, Meck and Williams. This is an open-access article subject to a non-exclusive license between the authors and Frontiers Media $S A$, which permits use, distribution and reproduction in other forums, provided the original authors and source are credited and other Frontiers conditions are complied with. 